\title{
Machinery, regulation and
} pathophysiological implications of autophagosome maturation

Yan G. Zhao $\mathbb{B}^{\prime}$, Patrice Codogno $\mathbb{D}^{2 凶}$ and Hong Zhang $\mathbb{1}^{3,4} \bowtie$

Abstract | Autophagy is a versatile degradation system for maintaining cellular homeostasis whereby cytosolic materials are sequestered in a double-membrane autophagosome and subsequently delivered to lysosomes, where they are broken down. In multicellular organisms, newly formed autophagosomes undergo a process called 'maturation', in which they fuse with vesicles originating from endolysosomal compartments, including early/late endosomes and lysosomes, to form amphisomes, which eventually become degradative autolysosomes. This fusion process requires the concerted actions of multiple regulators of membrane dynamics, including SNAREs, tethering proteins and RAB GTPases, and also transport of autophagosomes and late endosomes/lysosomes towards each other. Multiple mechanisms modulate autophagosome maturation, including post-translational modification of key components, spatial distribution of phosphoinositide lipid species on membranes, RAB protein dynamics, and biogenesis and function of lysosomes. Nutrient status and various stresses integrate into the autophagosome maturation machinery to coordinate the progression of autophagic flux. Impaired autophagosome maturation is linked to the pathogenesis of various human diseases, including neurodegenerative disorders, cancer and myopathies. Furthermore, invading pathogens exploit various strategies to block autophagosome maturation, thus evading destruction and even subverting autophagic vacuoles (autophagosomes, amphisomes and autolysosomes) for survival, growth and/or release. Here, we discuss the recent progress in our understanding of the machinery and regulation of autophagosome maturation, the relevance of these mechanisms to human pathophysiology and how they are harnessed by pathogens for their benefit. We also provide perspectives on targeting autophagosome maturation therapeutically.

凶e-mail: patrice.codogno@ inserm.fr; hongzhang@ ibp.ac.cn
Cells in multicellular organisms constantly experience diverse stresses, including protein misfolding, organelle damage, scarcity of nutrients/energy and invasion by pathogens. One mechanism exploited by cells to combat stresses is the lysosome-mediated degradation of intracellular components via autophagy. Autophagy involves the formation of an isolation membrane (or phagophore), which further expands and closes to form the double-membrane autophagosome ${ }^{1-3}$. The mechanism of autophagosome formation was excellently reviewed recently ${ }^{3}$. Various cellular materials can be sequestered in autophagosomes, including unselected cytosolic material or selected cargos such as protein aggregates, damaged organelles and pathogens. Following autophagosome closure via membrane abscission, cargos are delivered to the vacuole (yeasts and plants) or to lysosomes (animal cells) (for an overview of the autophagy process see ${ }^{4-8}$; BOX 1). After degradation of the autophagic cargo, the digested content in the autolysosomes is released and lysosomes are re-formed to sustain the autophagic flux (BOX 2). A series of proteins encoded by ATG (autophagy-related) genes and EPG (ectopic PGL granules) genes - identified mainly from genetic screens in Saccharomyces cerevisiae and Caenorhabditis elegans, respectively - act at different membrane remodelling steps for autophagosome formation and maturation $^{3,4,9,10}$.

In yeast, autophagosomes are formed in the vicinity of and directly fuse with the much larger vacuole, whereas in multicellular organisms, newly formed autophagosomes fuse with different endolysosomal vesicles such as early/late endosomes and lysosomes to form non-degradative, single-membrane structures called 'amphisomes', which gradually acquire 


\section{V-ATPase}

A proton pump, driven by ATP hydrolysis, which transports protons into the lumen of membrane compartments for their acidification. V-ATPases are multisubunit complexes, containing $\mathrm{V} 1$ and $\mathrm{V} 0$ sectors

SNARE (soluble

$\mathrm{N}$-ethylmaleimide-sensitive factor attachment protein receptor) complexes Complexes consisting of the $\mathrm{Qa}, \mathrm{Qb}, \mathrm{Qc}$ and $\mathrm{R}$ SNARE proteins in opposing membranes. The SNARE motifs of these proteins are assembled into a heterooligomeric, four-helix bundle to drive membrane fusion.

\section{Tethers}

Proteins or protein complexes that link the transported vesicles to target membranes and also promote the assembly of the trans-SNARE complex.

Phosphoinositides

Phosphorylated derivatives of phosphatidylinositol that define membrane identity and participate in various signalling and membrane trafficking processes.

\section{RAB proteins}

A subgroup of small GTPases that dynamically associate with membrane-bound compartments driven by GTP-GDP cycling to regulate dynamics, such as vesicle transport, fusion and positioning.

Multivesicular bodies Endocytic vesicles formed by invagination of the endosomal membrane to form membranebound intraluminal vesicles. They subsequently undergo lysosomal degradation or extracellular release. multiple aspects of membrane

degradative properties through the accumulation of hydrolases. Maturation also involves autophagosome acidification mediated by V-ATPase, which is required for the activity of degradative enzymes ${ }^{9-11}$. Fusion of autophagosomes with endosomes/lysosomes requires the concerted actions of cognate SNARE (soluble $\mathrm{N}$-ethylmaleimide-sensitive factor attachment protein receptor) complexes, tethers, phosphoinositides and RAB proteins ${ }^{11}$. Bidirectional microtubule-based transport of autophagic vacuoles (a term encompassing autophagosomes, amphisomes and autolysosomes) and late endosomes/lysosomes between the perinuclear space and periphery of cells maximizes the frequency of encounters and fusion efficiency ${ }^{12}$.

Recent studies revealed that the machinery mediating autophagosome maturation, such as SNARE complex assembly, tether recruitment and RAB protein dynamics, is tightly controlled and highly responsive to nutrient availability and stress conditions ${ }^{11}$. Disturbing these processes can cause accumulation of damaged organelles and toxic protein aggregates, and may also hamper endocytic trafficking. Accordingly, impaired autophagosome maturation is associated with the pathogenesis of various human disorders, including neurodegenerative diseases, cancer and myopathies ${ }^{11,13}$. Furthermore, invading viruses and bacteria also widely target maturation of autophagosomes to block their degradative capability. Viruses and bacteria thereby evade destruction and also accumulate autophagic vacuoles for their survival, replication and release ${ }^{14-17}$. Notably, unlike impairment of genes essential for autophagosome formation, loss of function of genes acting at autophagosome maturation usually causes a weaker defect and gradual accumulation of autophagic vacuoles. More importantly, in some cases, such defects can be suppressed by promoting the activities of partially redundant factors or mechanisms. For example, facilitating the recruitment of one tethering factor would alleviate the defect caused by depletion of another. This offers opportunities to ameliorate the defects of autophagosome maturation in relevant human diseases.

In this Review, we outline the most recent progress in our understanding of the molecular machinery that drives and regulates autophagosome maturation. We further discuss the causative link between impaired autophagosome maturation and the pathogenesis of neurodegeneration, muscle diseases and cancer. We also consider how viral proteins and bacterial effectors block autophagosome maturation for their own benefits. Finally, we offer insights into how autophagosome maturation can be therapeutically targeted to combat disease.

\footnotetext{
Author addresses

${ }^{1}$ Department of Biology, School of Life Sciences, Southern University of Science and Technology, Shenzhen, People's Republic of China.

'Institut Necker-Enfants Malades, INSERM U1151-CNRS UMR 8253, Université de Paris, Paris, France.

${ }^{3}$ National Laboratory of Biomacromolecules, CAS Center for Excellence in Biomacromolecules, Institute of Biophysics, Chinese Academy of Sciences, Beijing, People's Republic of China.

${ }^{4}$ College of Life Sciences, University of Chinese Academy of Sciences, Beijing,

People's Republic of China.
}

\section{Machinery for autophagosome fusion}

Maturation of autophagosomes requires their fusion with functional endolysosomal compartments such as early endosomes, late endosomes/multivesicular bodies and lysosomes. Accordingly, disturbances in biogenesis of these endolysosomal compartments - such as those caused by the loss of function of subunits of the ESCRT (endosomal sorting complexes required for transport) complex and COPI vesicles - cause accumulation of non-degradative autophagic vacuoles ${ }^{18,19}$. Fusion of autophagosomes with late endosomes/lysosomes involves the concerted action of RABs, tethers and the SNARE complex. Membrane fusion is driven by the assembly of a trans-SNARE complex composed of Qa, Qb, Qc and R SNAREs $^{20}$. Tethering factors, which are recruited to target membranes and/or fusing vesicles by the active form of small RAB GTPases, phosphoinositides and SNARE proteins, facilitate the initial capture of vesicles and subsequent formation of the trans-SNARE complex ${ }^{21,22}$. Autophagic vacuoles are decorated with lipidated (phosphatidylethanolamine-conjugated) ubiquitin-like autophagy proteins related to yeast Atg8. These proteins, in the LC3 subfamily or the GABARAP subfamily, also help to recruit other factors such as tethers and RAB guanine nucleotide exchange factors (GEFs) for maturation $^{23,24}$. LC3 is a widely used autophagic marker that labels autophagic structures at different stages of biogenesis, and, together with markers for late endosomes/ lysosomes, it accumulates on amphisomes during maturation. Of note, in studies using LC3 to label autophagic structures after closure (which can be differentiated from isolation membranes by co-labelling with markers for other autophagy proteins or use of the HaloTag-LC3 method $^{6,25}$ ), double-membrane autophagosomes cannot be distinguished from single-membrane amphisomes and autolysosomes. Hence, LC3-marked structures after closure should be referred to as 'autophagic vacuoles' unless the structure has been unambiguously demonstrated.

The role of SNARE complexes. SNARE complexes formed by autophagosomal membrane-localized STX17 (Qa), SNAP29 (Qbc) and late endosomal/lysosomallocalized VAMP8 (Vamp7 in flies and VAMP-7 in worms), or by the autophagosomal YKT6, SNAP29 and late endosomal/lysosomal-localized STX7, function partially redundantly to drive fusion of autophagosomes with late endosomes/lysosomes ${ }^{26-28}$ (FIG. 1a). Targeting of STX17 to forming autophagosomes accompanies autophagosome closure ${ }^{29}$. The small guanosine triphosphatase IRGM facilitates translocation of STX17 to autophagosomes by interacting with STX17 and ATG8-like proteins on the autophagosomal membrane ${ }^{30}$. SNAP29 is recruited by interacting with other SNAREs. STX17-SNAP29 complex assembly is promoted by the autophagosome-associated autophagy protein ATG14, which directly interacts with STX17 $\left(\right.$ REF. $^{31}$ ) (FIG. 1a). Different SNARE complexes may act at different steps of autophagosome maturation or drive fusion of autophagic vacuoles with different sets of late endosomes/lysosomes ${ }^{27,32}$. Postfusion SNAREs disassemble and return to their donor compartments to 


\section{Box 1 | Overview of the autophagy pathway}

Autophagy, a lysosome-mediated degradation system, is an evolutionarily conserved pathway for recycling cellular content and for removing aggregated proteins, damaged organelles and invading pathogens (for example, viruses and bacteria) to maintain normal cellular function and homeostasis. Autophagy occurs in a stepwise manner involving sequential membrane remodelling processes (see the figure). Upon induction, autophagy starts with the initiation and nucleation of a doublemembrane sac, known as the isolation membrane (or phagophore), in the cytosol $^{1-3}$. Vesicles carrying the multispanning membrane protein ATG9 have been suggested to serve as one of the membrane sources for isolation membrane initiation and nucleation. The isolation membrane then undergoes expansion to surround the cargos. Multiple membrane sources have been reported to contribute to isolation membrane growth, including the endoplasmic reticulum (ER), lipid droplets, the ER-Golgi intermediate compartment (ERGIC), the plasma membrane, ATG9 vesicles and COPII vesicles ${ }^{1-3,10}$. Isolation membrane expansion can also be mediated by lipid transfer from the ER and local fatty acid synthesis ${ }^{216,217}$. Once the autophagosome is fully closed, it fuses with late endosomes/lysosomes to form an amphisome/autolysosome. The sequestrated materials are broken down in degradative autolysosomes.

Genetic screens in yeast identified a group of conserved autophagy proteins, named 'Atg (autophagy-related) proteins', which function at different steps of autophagosome formation ${ }^{3,10,68}$. According to their function, these proteins can be divided into five groups. (1) The Atg1-Atg13-Atg17 kinase complex, whose mammalian counterpart is the ULK1-ATG13-FIP200 complex, is first recruited to the autophagosome formation site and triggers the nucleation of isolation membranes. (2) The phosphatidylinositol 3-phosphate (Ptdlns3P) kinase complex, consisting of
Vps34, Atg6 (mammalian beclin 1 homologue) and Atg14, is responsible for local production of Ptdlns3P to recruit downstream effectors. (3) The Atg12 conjugation system, comprising the E1 enzyme Atg7 and the E2 enzyme Atg10, catalyses conjugation of ubiquitin-like protein Atg12 with Atg5. The Atg12-Atg5 conjugate further interacts with Atg16. (4) The Atg8 conjugation system, comprising Atg7 and the E2 enzyme Atg3, catalyses conjugation of ubiquitin-like protein Atg8 to phosphatidylethanolamine. The Atg12 and Atg8 conjugation systems function at multiple steps of autophagy, including isolation membrane expansion, isolation membrane closure and cargo recognition in selective autophagy. (5) The Atg2-Atg18 complex and the transmembrane protein Atg9 are possibly involved in tethering the ER with the isolation membrane to transfer glycerophospholipids for isolation membrane growth. By recruiting different effectors, Ptdlns3P has multiple functions in autophagy, including initiation and expansion of the isolation membrane and also fusion of autophagosomes with the vacuole ${ }^{11}$.

Autophagy in multicellular organisms contains steps that are absent in yeast autophagy, including the recruitment of autophagy proteins to the autophagosome formation sites on the ER, the formation of complex ER-isolation membrane contacts during isolation membrane expansion, and autophagosome maturation. Genetic screens in Caenorhabditis elegans identified multiple metazoan-specific autophagy proteins, that is, EPG (ectopic PGL granules) proteins. These proteins act at steps unique to autophagy in multicellular organisms. EPG-3 (known as VMP1 in mammals) is required for establishing dynamic ER-isolation membrane contacts ${ }^{157}$, while EPG-5 and WDR45/WDR45B mediate autophagosome-lysosome fusion ${ }^{25,40}$. Thus, ATG and EPG proteins act together to complete the more complex mammalian autophagy process.

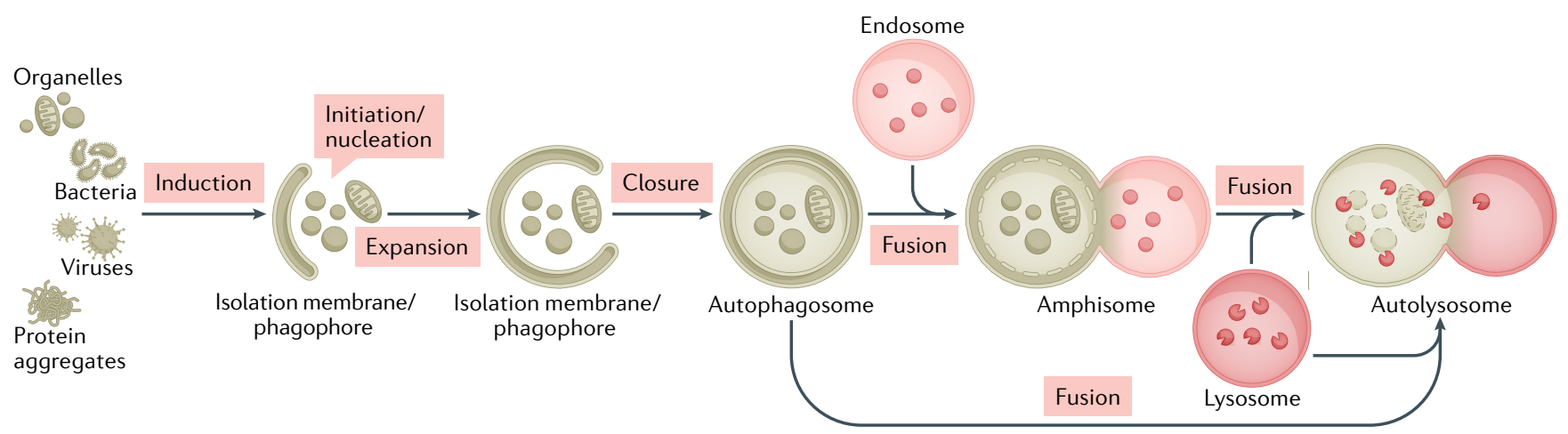

COPI vesicles

Coat protein I (COPI)-coated

vesicles mediating the retrieval of proteins and lipids from the Golgi apparatus to the endoplasmic reticulum and also transport between Golgi apparatus cisternae.

LC3 subfamily

A subfamily of Atg8

homologues containing

LC3A, LC3B and LC3C.

GABARAP subfamily

A subfamily of Atg8

homologues containing

GABARAP, GABARAPL 1

and GABARAPL2. maintain intracellular membrane identity and to prepare for new rounds of fusion ${ }^{20}$. The molecular mechanisms driving SNARE retrieval from autolysosomes have yet to be determined. It has been shown that release of STX17 from autolysosomes coincides with collapse of the autophagosomal inner membrane due to breakdown by lysosomal enzymes ${ }^{29}$.

The role of tethering factors. Both multisubunit tethering complexes and large individual tether proteins participate in the initial capture of autophagosomes and/or amphisomes and promote fusion efficiency and specificity with early/late endosomes and lysosomes but not with other vesicles such as recycling endosomes or secretory vesicles during autophagosome maturation (FIG. 1 b).

The HOPS (homotypic fusion and protein sorting) complex is a prominent tethering complex that promotes assembly of the trans-SNARE complex, acts as a GEF for RAB7 and functions as a SNARE chaperone to facilitate fusion of autophagosomes with late endosomes and lysosomes $22,33,34$. The HOPS complex can be targeted to both autophagosomes and late endosomes/lysosomes to promote fusion ${ }^{33,34}$. Targeting of the HOPS complex to late endosomes/lysosomes is mediated by its binding to RAB7 and phosphoinositides such as phosphatidylinositol 3-phosphate (PtdIns3P) ${ }^{35}$, while multiple mechanisms facilitate its translocation from the cytosol to autophagosomes, including interaction with STX17 (REFS ${ }^{33,34}$ ), active RAB7 (REFS ${ }^{36,37}$ ), GABARAPs ${ }^{38}$ or large tether proteins (such as PLEKHM1) described below (FIG. 1 b).

EPG5 is an evolutionarily conserved large tether protein for autophagosome maturation ${ }^{39,40}$. It is a RAB7 effector and translocates from the cytosol to late endosomes/lysosomes by interacting with RAB7 (REF. ${ }^{40}$ ) (FIG. 1 b). During autophagosome maturation, EPG5 captures autophagosomes/amphisomes via binding to LC3 and promotes assembly of the STX17-SNAP29-VAMP8 complex ${ }^{40}$. EPG5 depletion causes accumulation of autophagosomes, amphisomes and non-degradative 


\section{Guanine nucleotide \\ exchange factors \\ (GEFs). Factors that catalyse \\ the conversion of the \\ GDP-bound inactive form \\ of small GTPase proteins to \\ their GTP-bound active form \\ Recycling endosomes Endosomal compartments for recycling materials internalized by endocytosis back to the cell surface to maintain the composition of the plasma membrane.}

HOPS (homotypic fusion and protein sorting) complex

A multisubunit seahorseshaped complex consisting of VPS41, VPS39, VPS18, VPS33A, VPS11 and VPS16. It facilitates fusion events involving late endosomes and lysosomes.

SNARE chaperone A protein chaperone such as Sec1/Munc 18 (SM) proteins that binds to individual SNAREs or assembly intermediates. It ensures the fast and accurate assembly of trans-SNARE complexes to drive efficient membrane fusion.

\section{Box 2 | Degradation, catabolite export and lysosome re-formation}

After autophagosome maturation, the inner membrane disintegrates and is degraded together with sequestrated materials in acidic autolysosomes by hydrolases ${ }^{218}$. Specific hydrolases are involved in the degradation of different macromolecules, such as proteins, nucleic acids, lipids and carbohydrates. The glycosylated integral membrane protein Atg15 is a lipase essential for disintegration of inner membranes ${ }^{219}$. In Caenorhabditis elegans, the T2 family endoribonuclease RNST-2 degrades ribosomal RNAs that are delivered to lysosomes via autophagy ${ }^{220}$. The lysosomal degradation products are exported to the cytosol via lysosomal efflux transporters, such as the lysosomal lysine/ arginine transporter LAAT-1 and the sugar transporter Spinster ${ }^{221,222}$. The exported catabolites provide building blocks (for example, amino acids, nucleotides and monosaccharides) or materials for energy production to maintain cellular homeostasis. For example, autophagy-dependent ribosomal RNA degradation is essential for maintaining nucleotide homeostasis during C. elegans development ${ }^{220}$. Defective lysosomal degradation or catabolite export from lysosomes leads to impaired lysosomal function and accumulation of undigested materials or digested catabolites.

Upon release of the digestion products, lysosomes are regenerated from autolysosomes, a process known as autophagic lysosome re-formation (ALR), to maintain lysosome homeostasis ${ }^{223}$. ALR involves clathrin-mediated budding of tubular structures from autolysosomes, KIF5B-driven extension of membrane tubules along microtubules, dynamin 2-mediated scission of protolysosomes devoid of lysosomal contents and subsequent maturation of protolysosomes to functional lysosomes ${ }^{224}$. During prolonged starvation-induced autophagy, ALR is triggered by mTOR reactivation, which is normally repressed under starvation. This requires degradation of autophagic cargo and release of degraded products, among which are amino acids - the primary activators of mTOR. Defective autophagic degradation or export of the degradation products from the autolysosome, such as in cells derived from patients with lysosome storage diseases, inhibits mTOR reactivation and ALR, resulting in accumulation of enlarged autolysosomes ${ }^{223}$. The activity of the lysosomal channel protein TRPML1 is also involved in autolysosome re-formation ${ }^{225}$. Lysosomal regeneration at the end of autophagic flux is critical for maintaining the lysosome pool and thus sustained autophagy. maturation requires retrograde transport of autophagic vacuoles and lysosomes driven by the microtubule motor protein dynein, as well as their anterograde transport mediated by kinesin motors ${ }^{12,46,47}$, which allow the two compartments to meet and fuse.

\section{Machinery mediating transport of autophagic vacuoles} and lysosomes. Anterograde transport of lysosomes enables them to fuse with peripheral autophagosomes. Anterograde transport is mediated by the late endosomal/ lysosomal-associated multisubunit BORC complex, which recruits the small GTPase ARL8 to promote ARL8dependent coupling to kinesin motors ${ }^{12}$ (FIG. 2). ARL8 directly interacts with kinesin 3, or interacts with its effector SKIP, which in turn couples to kinesin $1\left(\right.$ REF. $\left.^{48}\right)$. Depletion of BORC complex decreases the fusion of peripheral autophagosomes with lysosomes ${ }^{49}$. The RAB7 effector FYCO1 also acts as an adaptor of kinesin 1 to mediate anterograde transport of late endosomes/ lysosomes, and of autophagic vacuoles through its binding to LC3 and PtdIns3 $3 \mathrm{P}^{50}$ (FIG. 2).

The recruitment of the dynein-dynactin motor complex to late endosomes/lysosomes and autophagic vacuoles for retrograde transport is mediated by RAB7 and its effectors RILP and ORP1L ${ }^{12}$ (FIG. 2). RILP interacts with the p150 subunit of the dynein-dynactin motor complex ${ }^{12}$, whereas ORP1L promotes the binding of the dynein complex with late endosomal/lysosomal membrane-associated $\beta$ III spectrin ${ }^{51}$. In addition, ORP1L can sense and couple cholesterol levels with transport of lysosomes and autophagic vacuoles (FIG. 2). Under low-cholesterol conditions, ORP1L interacts with the endoplasmic reticulum (ER) protein VAPA to form contacts between the ER and lysosomes/ autophagic vacuoles; the dynein motor then disassociates from RAB7-RILP, causing dispersal of these organelles $^{43,52}$. In Niemann-Pick type C disease and other lysosome storage diseases (LSDs), accumulation of cholesterol in the late endosomal/lysosomal compartments results in their perinuclear clustering and a defect in autophagosome maturation ${ }^{52}$.

Transport of autophagic vacuoles and late endosomes/lysosomes is tightly coordinated with recruitment of tethering factors. Specifically, the HOPS complex can be recruited by ARL8 $\left(\mathrm{REF}^{49}\right.$ ) or by the ORP1LRAB7-RILP complex, which occurs either directly or via PLEKHM1 (REF. $\left.{ }^{53}\right)$. This allows coupling of anterograde and retrograde transport and fusion events ${ }^{53}$.

Directional transport of autophagic vacuoles is particularly prominent in polarized neuronal cells, where autophagosomes formed at the distal synaptic termini undergo long-range retrograde transport to the soma ${ }^{54,55}$. Maturation of autophagosomes is tightly linked with their dynein-driven transport to the soma, which requires coordinated actions of scaffolding proteins, including JNK-interacting protein 1 (JIP1), HTT-associated protein 1 (HAP1) and JIP3 $\left(\right.$ REFS $^{56,57}$ ). These factors bridge autophagic vacuoles with the dynein-dynactin complex, and their actions depend on location and autophagosomal maturity ${ }^{56,57}$. JIP1 acts in the distal portion of the axon, HAP1 functions in the mid-axon, while JIP3 primarily controls the motility of 


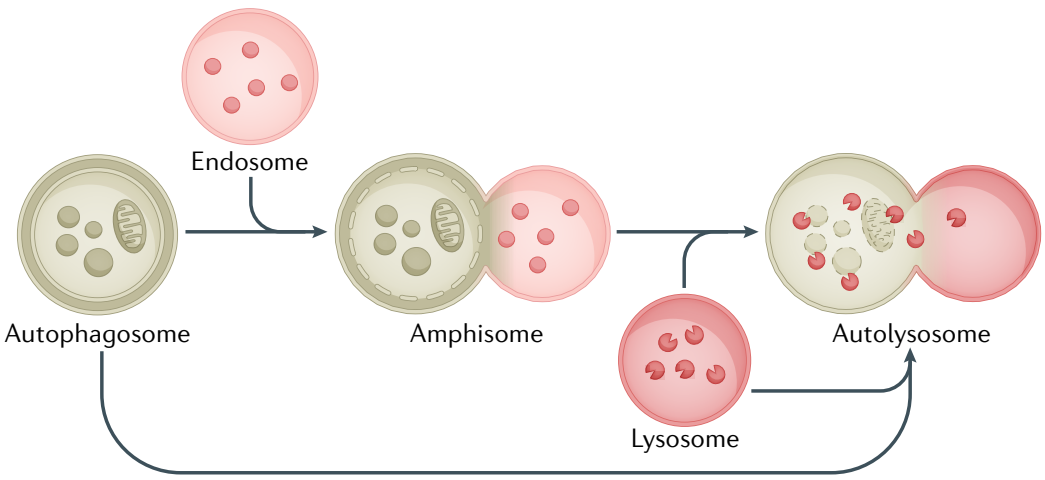

a

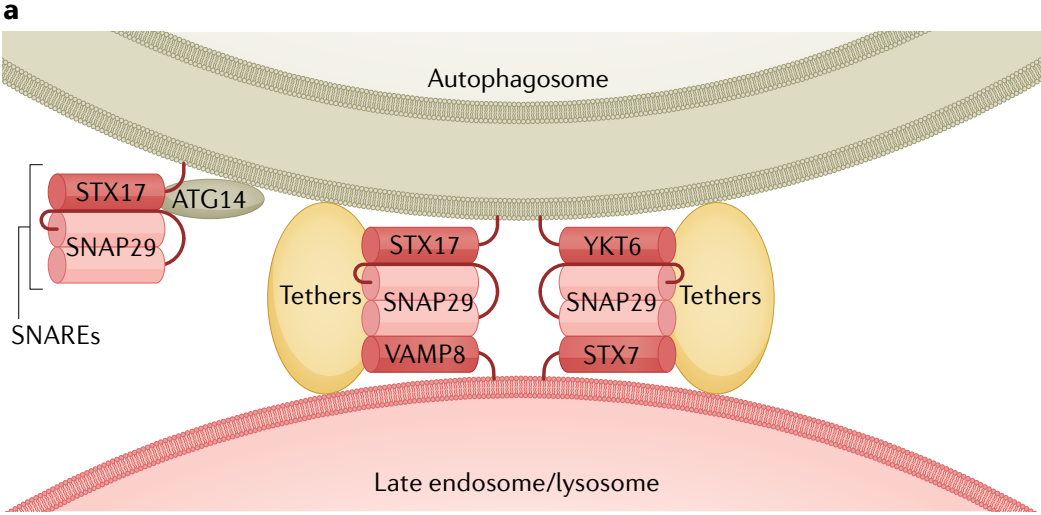

b

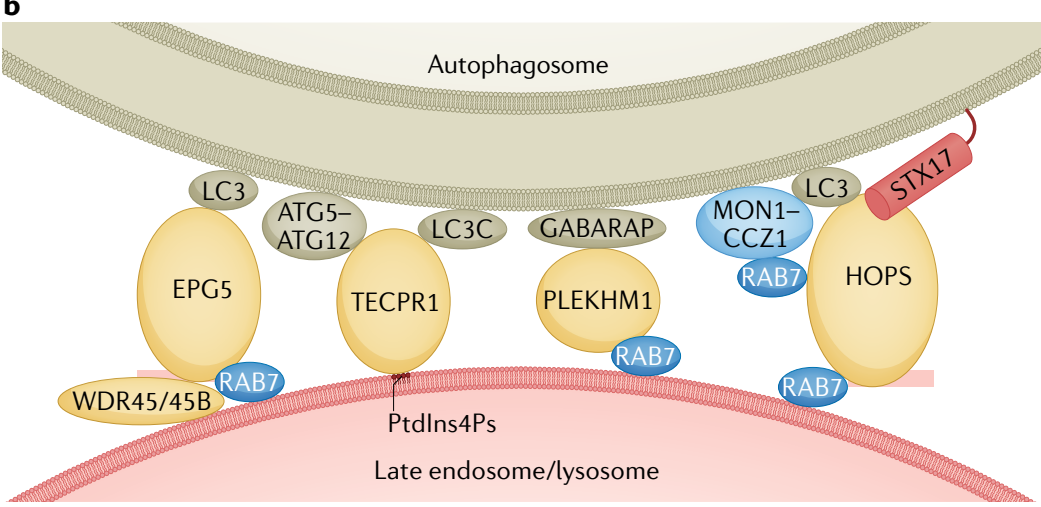

$\beta$-Propeller proteins

A family of proteins with 4-12 repeats of a $\beta$-stranded blade that function as structural scaffolds for ligand binding, enzymatic activity and assembly of multiple protein complexes.

Pleckstrin homology (PH) domain

A protein domain that binds phosphatidylinositol lipids (such as phosphatidylinositol 4,5-bisphosphate) and proteins with high affinity and specificity. It functions in vesicle trafficking, cellular signalling and cytoskeletal remodelling. maturer autophagic vacuoles in the proximal portion of the axon ${ }^{57}$.

\section{Regulation of autophagosome maturation}

The machinery governing autophagosome maturation is dynamically regulated to allow adaptation of autophagic flux to the needs of the cell and to enable integration of autophagic degradation with external inputs. This involves regulation of the membrane recruitment of the key components mediating fusion events as well as transcriptional regulation, which occurs predominantly via the MiT/TFE family transcription factors TFEB and TFE3. During autophagosome biogenesis, various stresses and signalling pathways can modulate the initiation of autophagosome formation via multiple mechanisms, such as by regulating the kinase activity, stability and assembly of the Atg 1 complex (ULK1 complex in mammals) and the Vps34 Ptdlns3P kinase complex ${ }^{58-62}$. mTORC1, an evolutionarily conserved signalling hub that senses nutrient status and growth factor signals,
Fig. 1 SNAREs, tethers and RAB proteins mediate autophagosome maturation. a | Fusion between autophagosomes and late endosomes/lysosomes is mediated by two sets of SNARE complexes: the autophagosomal Qa SNARE STX17, the Qbc SNARE SNAP29 and the endolysosomal R SNARE VAMP8 (centre left); and the autophagosomal R SNARE YKT6, SNAP29 and the endolysosomal Qa SNARE STX7 (centre right). The unique hairpin structure formed by the two transmembrane domains of STX17 and the amino-terminal longin domain of YKT6 are involved in their autophagosomal recruitment ${ }^{26,27}$. The autophagosomeassociated autophagy protein ATG14 interacts with STX17 to promote the assembly of the STX17-SNAP29 subcomplex (left). The assembly and function of both complexes are facilitated by tether proteins. $\mathbf{b}$ | Multiple tether proteins are involved in fusion of autophagosomes with late endosomes/lysosomes. EPG5 is targeted to autophagosomes and lysosomes by binding to LC3 and RAB7, respectively. In neural cells, WDR45 and WDR45B facilitate the lysosomal localization of EPG5. TECPR1 interacts with LC3C and ATG5-ATG12 on autophagosomes and phosphatidylinositol 4-phosphate (Ptdlns4P) on lysosomes. RAB7 binds to the HOPS complex and PLEKHM1. On the autophagosome side, the HOPS complex binds to STX17 and LC 3 proteins, and PLEKHM1 binds to GABARAPs. The active GTP-bound RAB7 associates with the membrane. Localization of RAB7 on autophagic vacuoles is facilitated by the LC3-binding MON1-CCZ1 guanine nucleotide exchange factor complex $^{36,37}$.

plays a key role here. Nutrient status and other stresses also integrate into the autophagosome maturation machinery to add another level of control of autophagic flux. It is also noteworthy that the machinery governing autophagosome maturation is heterogeneous and demonstrates at least partial redundancy, which may allow flexible regulation of autophagic flux in different cell types.

Trafficking and post-translational modification of SNARE proteins. SNARE proteins dynamically move between distinct membrane compartments ${ }^{20}$. Upon starvation, RAB21, an endosomal RAB protein, is activated by its GEF MTMR13, which further promotes the translocation of plasma membrane-localized VAMP8 to late endosomes/lysosomes ${ }^{63}$ (FIG. 3a). SNARE protein dynamics is required for efficient fusion of the endolysosomal structures with autophagic vacuoles as evidenced by LSDs such as multiple sulfatase deficiency and mucopolysaccharidosis type IIIA, where SNAREs are sequestered in cholesterol-enriched regions of endolysosomal membranes and locked in assembled complexes ${ }^{64}$. Therefore, disassembly of postfusion SNARE complexes and their sorting and recycling back to target membranes is impaired, and, consequently, fusion of lysosomes with endocytic and autophagic vesicles is reduced ${ }^{64}$.

The SNARE activity of STX17 is regulated by acetylation of its SNARE domain, a modification controlled by the histone acetyltransferase CREBBP/CBP and the deacetylase HDAC2 (REF. ${ }^{65}$ ) (FIG. 3a). Starvation or mTORC1 inhibition inactivates CREBBP, while promoting the deacetylation of STX17. Deacetylation of STX17 facilitates the assembly of the STX17-SNAP29-VAMP8 complex and also enhances its binding to the HOPS 
Retrograde transport Intracellular movement from the cell periphery towards the nucleus mediated by the dynein-dynactin complex Also known as centripetal movement or minus-end transport.

\section{Anterograde transport} Intracellular movement from the nucleus towards the cell periphery mediated by kinesin motors. Also known as centrifugal movement or plus-end transport.

\section{BORC complex}

A multisubunit complex that associates peripherally with the lysosomal membrane to regulate lysosomal positioning by recruiting ARL8. It comprises eight subunits: BLOS1, BLOS2, snapin, KXD1, myrlysin, lyspersin, diaskedin and MEF2BNB.

\section{ARL8}

A small ADP-ribosylation factor-like RAS family GTPase that mediates kinesin-driven lysosome transport, and also regulates lysosome fusion by recruiting the HOPS complex.

Lysosome storage diseases (LSDs). A group of inherited metabolic disorders characterized by abnormal storage of toxic materials. They result from deficiencies of lysosomal enzymes or transporters.

Atg 1 complex The Atg 1 complex consists of the protein kinase Atg1 together with Atg13, Atg17. Atg31 and Atg29. Activation of this complex triggers the initiation of autophagy.

Vps34 Ptdlns3P kinase complex

A complex, containing the phosphatidylinositol 3-phosphate (Ptdlns3P) kinase Vps34 together with Vps 15, Atg6 and Atg14, that generates Ptdlns3P at the autophagosome formation site to recruit downstream effectors for autophagosome formation.

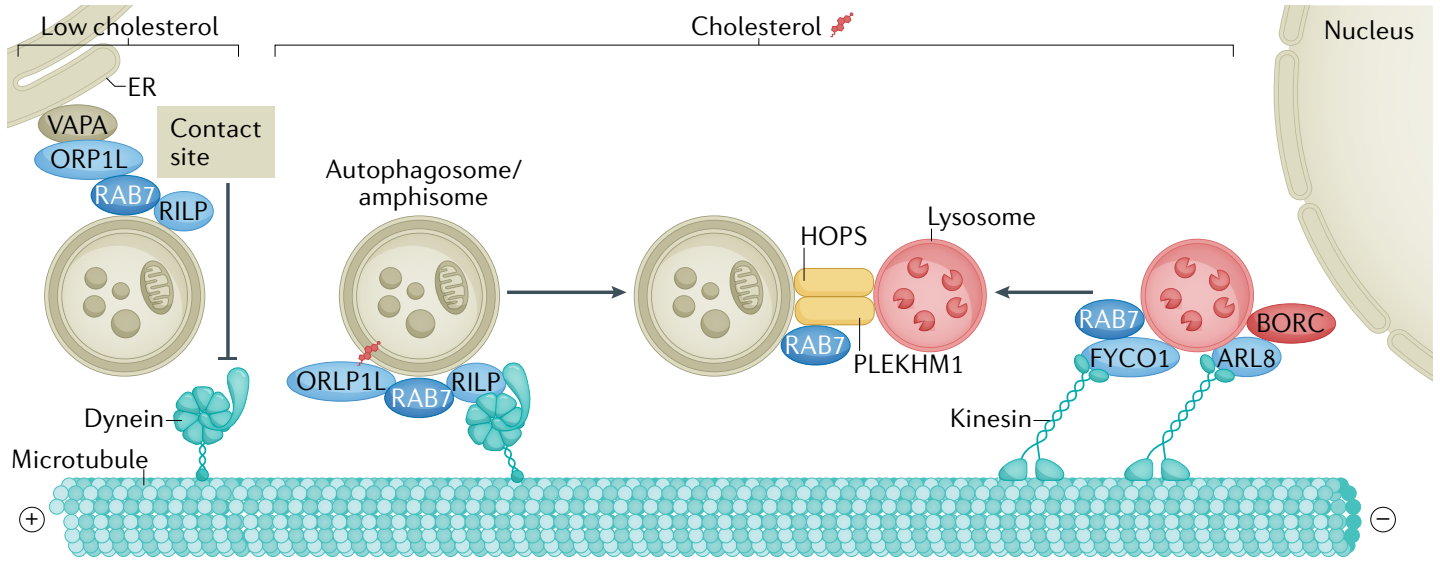

Fig. 2 | Coupling transport and fusion of autophagic vacuoles and late endosomes/lysosomes. Autophagosomes, which form throughout the cytoplasm, and lysosomes, which are localized mainly in the perinuclear region, undergo bidirectional movement on microtubules. The late endosome/lysosome-localized BORC-ARL8 complex and the FYCO1-RAB7 pair recruit kinesin motors for anterograde transport of late endosomes/lysosomes. The RAB7 effectors RILP and ORP1L promote the membrane association of the dynein-dynactin motor machinery to mediate the retrograde transport of autophagic vacuoles (as well as late endosomes/lysosomes (not shown)). When cholesterol levels are low, ORP1L interacts with the endoplasmic reticulum (ER) protein VAPA to form ER-autophagosome membrane contacts, which releases dynein-dynactin and prevents retrograde trafficking, thereby interfering with autophagosome maturation. During transport, the tethering factors HOPS complex and PLEKHM1 are concomitantly recruited to promote fusion processes (see FIG. 1).

complex, thus promoting autophagosome-lysosome fusion under stress conditions ${ }^{65}$ (FIG. 3a).

SNAP29 contains two antiparallel helix bundles and is the keySNARE for autophagosome maturation ${ }^{26,27}$. SNAP29 is post-translationally modified via O-GIcNAcylation at multiple serine/threonine residues, a process catalysed by O-linked $\beta$ - $N$-acetylglucosamine $(O-G l c N A c)$ transferase $(\mathrm{OGT})^{66}$. O-GlcNAcylation of SNAP29 attenuates SNARE complex assembly (FIG. 3a). OGT knockdown or expression of O-GlcNAcylation-defective SNAP29 facilitates the formation of SNAP29-containing SNARE complexes and promotes autophagic flux ${ }^{66}$. Levels of UDP-GlcNAc, the donor for $O$-GlcNAc addition, are responsive to the availability of glucose, fatty acids, uridine and glutamine ${ }^{67}$. The SNAP29 O-GlcNAcylation level is reduced by starvation in mammalian cells and worms ${ }^{66}$, thus integrating nutrient status with autophagosome maturation.

Regulation of HOPS complex recruitment. During autophagosome biogenesis, the VPS34-beclin 1-ATG14 complex (PI3K complex I) generates PtdIns3P on the $\mathrm{ER}$ and/or isolation membrane to recruit effectors such as ATG18 for autophagosome initiation ${ }^{3,68}$. Steady levels of PtdIns3P on autophagic vacuoles also facilitate autophagosome maturation by recruiting the tethering factors HOPS complex (FIG. 3b). The VPS34-beclin 1-UVRAG complex (PI3K complex II), which is known for production of PtdIns3P on endosomes ${ }^{69}$, appears to mediate the generation of PtdIns3P on autophagic vacuoles as well ${ }^{70}$. Pacer (protein associated with UVRAG as autophagy enhancer), which is recruited to autophagic vacuoles by binding STX17 and phosphoinositides, is involved in targeting the VPS34-beclin 1-UVRAG complex $^{71}$. Pacer and UVRAG also recruit the HOPS complex $^{71,72}$ (FIC. 3b). Rubicon, a RAB7 effector, which interacts with beclin 1 and associates with VPS34 complexes, antagonizes UVRAG function and negatively regulates autophagosome maturation ${ }^{70,73,74}$. The UVRAG-Rubicon interaction is enhanced by mTORC1mediated phosphorylation of UVRAG ${ }^{75}$, while active GTP-bound RAB7 and Pacer compete with Rubicon to release UVRAG, thus recruiting the HOPS complex and stimulating VPS34 activity under starvation ${ }^{71}$ (FIG. 3b). However, the role of UVRAG in autophagosome maturation is debated, as it is dispensable for autophagosomelysosome fusion in fly fat cells and in certain mammalian cells ${ }^{33,34}$.

In addition to PtdIns3P, PtdIns4P also participates in multiple steps of the autophagy pathway. During autophagosome biogenesis, PtdIns4P contributes to recruitment of the ULK1 complex subunit ATG13 to the autophagosome initiation site ${ }^{76}$. During maturation, GABARAPs recruit phosphatidylinositol 4-kinase IIa (PI4KIIa) to autophagosomes to generate PtdIns4P, thus facilitating autophagosome-lysosome fusion ${ }^{77}$. Accumulation of PtdIns4P on the endosomes/lysosomes also promotes fusion events. The spatiotemporal distribution of PtdIns4P is regulated by PI4Ks and the PtdIns4P phosphatase SAC1 $\left(\mathrm{REF}^{78}\right)$. SAC1 is an integral membrane protein that cycles between the ER and Golgi compartments via trafficking mediated by COPI vesicles and COPII vesicles. The ER-localized transmembrane protein SUSR2 (also known as TMEM39A) acts as an adaptor by simultaneously interacting with SAC1 and the COPII coat proteins SEC23 and SEC24 to facilitate ER-to-Golgi apparatus transport of SAC1 (REF. ${ }^{79}$ ). Retention of SAC1 on the ER in TMEM39Aknockdown cells increases the late endosomal/ lysosomal PtdIns4P level, which could result from an elevated PtdIns4P level in trans-Golgi network-derived anterograde vesicles and/or from an increased level of 
O-GlcNAcylation

A reversible post-translational modification in which a single O-linked $\mathrm{N}$-acetylglucosamine (O-GlcNAC) moiety is linked to serine/threonine residues of proteins. It is catalysed by $O$-GlcNAc transferase (OGT) and reversed by $O$-GlcNAcase (OGA)

COPII vesicles

Vesicles whose formation is driven by COPII coat proteins. COPIl vesicles transport proteins from the endoplasmic reticulum to the Golgi apparatus.

GTPase-activating protein (GAP). A protein that facilitates the GTPase activity of small GTPases resulting in hydrolysis of GTP to GDP and phosphate.

ER stress

A response elicited by accumulation of unfolded proteins in the endoplasmic reticulum (ER). It promotes several intracellular signal transduction pathways, collectively known as the unfolded protein response, to restore ER homeostasis.

Liquid-liquid phase separation

(LLPS). A process triggered by multivalent interactions of constituents, resulting in their concentration in a confined liquid-like compartment that stably coexists with the surrounding liquid milieu.

Mediator complex A multiprotein complex that interacts with transcription factors and RNA polymerase II and functions as a transcriptional co-activator. late endosomal/lysosomal PI4KIIa. Consequently, the recruitment of the HOPS complex, which binds strongly to PtdIns $4 \mathrm{P}^{35}$, is greatly facilitated and subsequently autophagosome maturation is enhanced ${ }^{79}$ (FIG. 3b).

Activity and dynamics of RAB7 proteins. RAB7 activity and dynamics - involving its switch between an active GTP-bound state and an inactive GDP-bound form on autophagic vesicles - are essential for progression of autophagic flux. RAB7 activity is negatively regulated by a GTPase-activating protein (GAP) called 'Armus' (also known as TBC1D2A), whose targeting is mediated via binding to LC3 on autophagic vacuoles and PtdIns3P generated by VPS34 on endosomes. Armus finely tunes the nucleotide cycle of RAB7 to promote autolysosome formation and acidification ${ }^{80,81}$ (FIG. 3b). Armus was also demonstrated to be regulated by the nutritional status of the cell via starvation-induced inactivation of the small GTPase RAC1, which competes with Armus for binding to LC3 on autophagic vacuoles ${ }^{81}$. Limiting Armus recruitment to target membranes leads to persistence of active RAB7 on autophagic vacuoles and endosomes, which may have multiple inhibitory effects on autophagosome maturation: it reduces the availability of mobile RAB7 required for endosome maturation into late endosomes/lysosomes; it maintains high activity of RAB7 effectors, such as Rubicon, whose aberrant activity may impede autophagosome maturation as described earlier; and it also impedes formation of intraluminal vesicles in multivesicular bodies ${ }^{80}-$ a process that is important for the endocytic pathway and hence the formation of amphisomes as well as for endosome maturation into late endosomes/lysosomes ${ }^{11,18,82}$. In addition to regulation by Armus, the conversion of endosomal PtdIns4P generated by PI4KIIa to phosphatidylinositol 4,5-bisphosphate also inactivates RAB7 and causes its disassociation from late endosomes and from PLEKHM1. This step also facilitates autophagosome maturation $^{83}$.

Regulation of TFEB/TFE3 activity by cytoplasm-tonucleus transport. TFEB and TFE3 are transcription factors with key functions in autophagy as they control expression of a network of genes involved in autophagosome formation, autophagosome maturation and lysosomal biogenesis ${ }^{84-87}$. Their activity is extensively regulated by phosphorylation status, controlled by various kinases and phosphatases, with phosphorylation of TFEB and TFE3 inhibiting their cytoplasm-to-nucleus trafficking ${ }^{86,88}$ (FIG. 3C). This regulation is coupled to nutrient availability via mTORC1. Under nutrientrich conditions, amino acids promote $\mathrm{mTORC} 1$ translocation to the lysosomal surface, where mTORC1 is activated. This lysosome surface-associated mTORC1 phosphorylates TFEB and TFE3, creating binding sites for the scaffold protein 14-3-3 for cytoplasmic retention, thereby preventing nuclear translocation and activity of these factors ${ }^{84,89}$. In addition to nutrients, various extracellular signals also regulate TFEB/TFE3. For example, it has been shown that glycogen synthase kinase $3 \beta$ (GSK $3 \beta)$ - which is inactivated by protein kinase C (PKC), which responds to several signal transduction cascades - phosphorylates TFEB to prevent it from translocating into the nucleus ${ }^{90}$. In response to various stresses, TFEB/TFE3 phosphorylation is inhibited and nuclear transport is facilitated ${ }^{84}$. TFEB and TFE3 are also actively dephosphorylated by the ubiquitous protein phosphatase $2 \mathrm{~A}$ (PP2A) and by calcineurin - a phosphatase activated by starvation-triggered lysosomal calcium release or ER stress ${ }^{91,92}$.

Regulation of TFEB activity via phase separation. Protein liquid-liquid phase separation (LLPS) is now an established mechanism for concentrating proteins in confined liquid-like compartments ${ }^{93-95}$. LLPS also compartmentalizes transcription factors, co-activators, the Mediator complex and RNA polymerase II into condensates for mediating gene expression ${ }^{96,97}$. It has been recently shown that TFEB undergoes LLPS and that phase-separated TFEB puncta colocalize with the Mediator complex and target mRNAs ${ }^{98}$. The nuclear protein inositol polyphosphate multikinase (IPMK) directly interacts with TFEB and chaperones it to inhibit its LLPS ${ }^{98}$ (FIG. 3c). IPMK knockout increases the formation of TFEB transcriptional condensates without altering TFEB phosphorylation or nuclear transport ${ }^{98}$. Consequently, IPMK knockout facilitates autophagosome maturation and promotes the maturation and degradation capability of lysosomes ${ }^{98}$.

Heterogeneity and redundancy of autophagosome maturation mechanisms. Overall, there is high variability in fusion events leading to mature, degradative autolysosomes. The process involves multiple, probably non-sequential, fusion processes, including fusion of autophagosomes with different endosomal and lysosomal structures, as well as various homotypic and heterotypic fusion events between the different types of autophagic vacuoles themselves ${ }^{11,46}$. This involves different tethers, which possess differential binding affinity for ATG8 members and thus mediate maturation of distinct populations of autophagosomes decorated with different ATG8-like proteins. Accordingly, different cell types, with distinct organization of endocytic vesicles, may require different tethers for autophagosome maturation. For example, PLEKHM1 depletion causes no autophagy defect in A549 lung adenocarcinoma cells and in C. elegans ${ }^{40,99}$. Loss of function of factors involved in autophagosome maturation may also result in accumulation of autophagic vacuoles at different stages of maturation in different cell types. Depletion of fly Rab2 causes accumulation of autophagosomes in muscle cells and amphisomes in larval fat cells ${ }^{100}$. To acquire degradative potential, autophagic vacuoles must be acidified efficiently, which is accomplished by further rounds of fusion with endosomes/lysosomes. Late endosomes/ lysosomes also show heterogeneity, with different surface $\mathrm{RAB}$ proteins and distinct resident hydrolytic enzymes. Hence, efficient autophagic degradation requires fusion of autophagic vacuoles with multiple late endosomes/ lysosomes ${ }^{11,46}$, which may require distinct SNAREs and tethers.

Notably, the functions of the multiple SNARE complexes and tethering factors are partially redundant, 
a

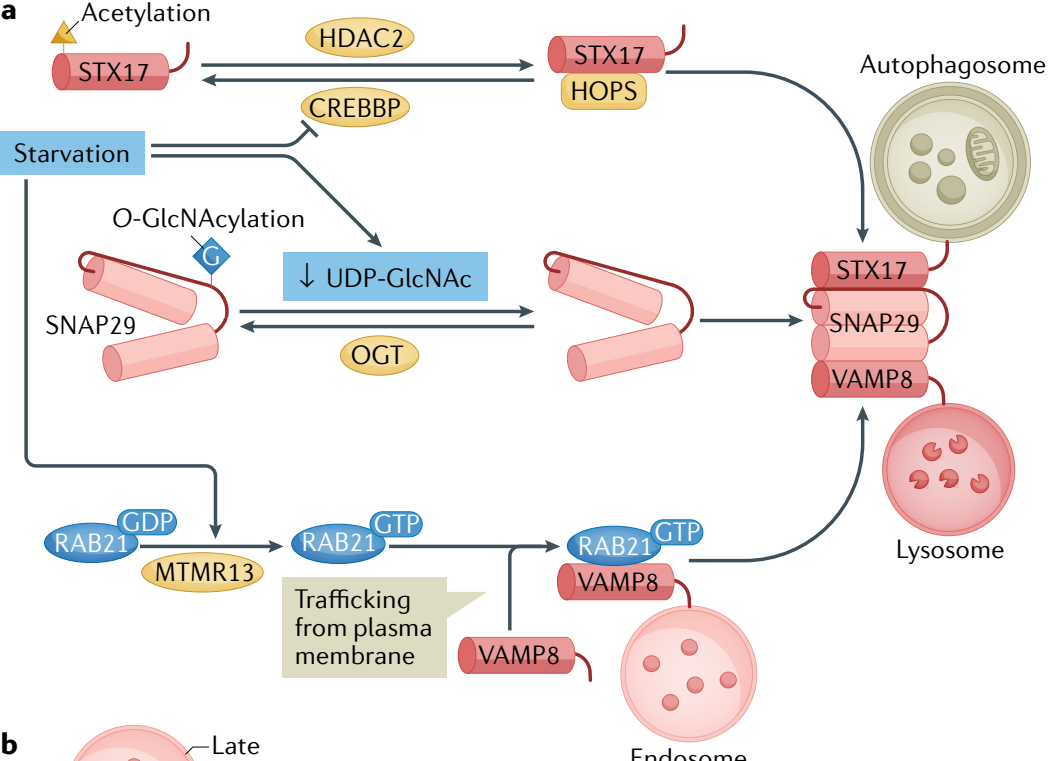

b

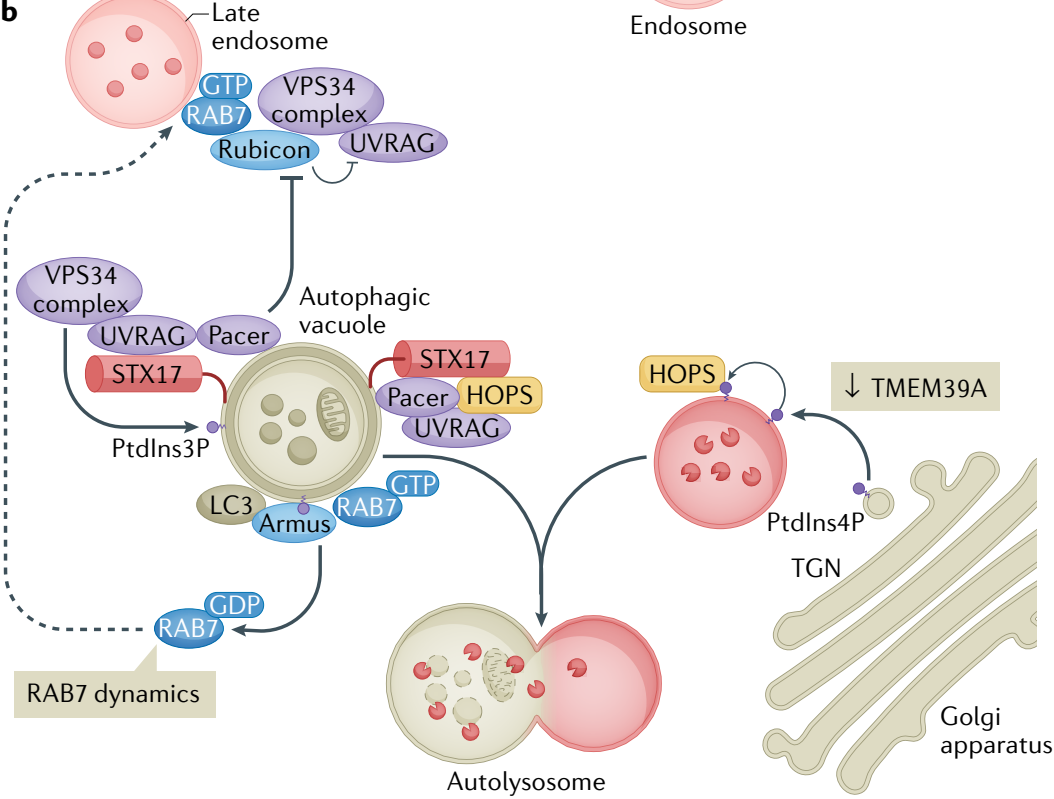

which, in some cases, means that impaired autophagosome maturation caused by depletion of one factor can be rescued by promotion of an alternative maturation mechanism. This is exemplified by characterization of suppressors of the autophagy defect associated with loss of EPG-5 activity in C. elegans. First, enhanced assembly of the STX17-SNAP29-VAMP8 complex achieved by reduced $O$-GlcNAcylation of SNAP29 suppresses the autophagy defect in epg-5-mutant worms, EPG5-deficient cells, cells depleted of VCP (also known as p97) - which is essential for maturation of ubiquitin cargo-containing autophagosomes (see also the next section) - and Wdr45-Wdr45b double-knockout cells $^{25,66}$. Second, the autophagy defect caused by EPG5 deficiency is also suppressed by enhancement of late endosomal/lysosomal recruitment of the HOPS complex in TMEM39A-knockdown cells or by elevation of lysosomal function and biogenesis by promoting TFEB activity via IPMK knockout ${ }^{79,98}$. Finally, defective autophagosome maturation in epg-5-mutant worms c

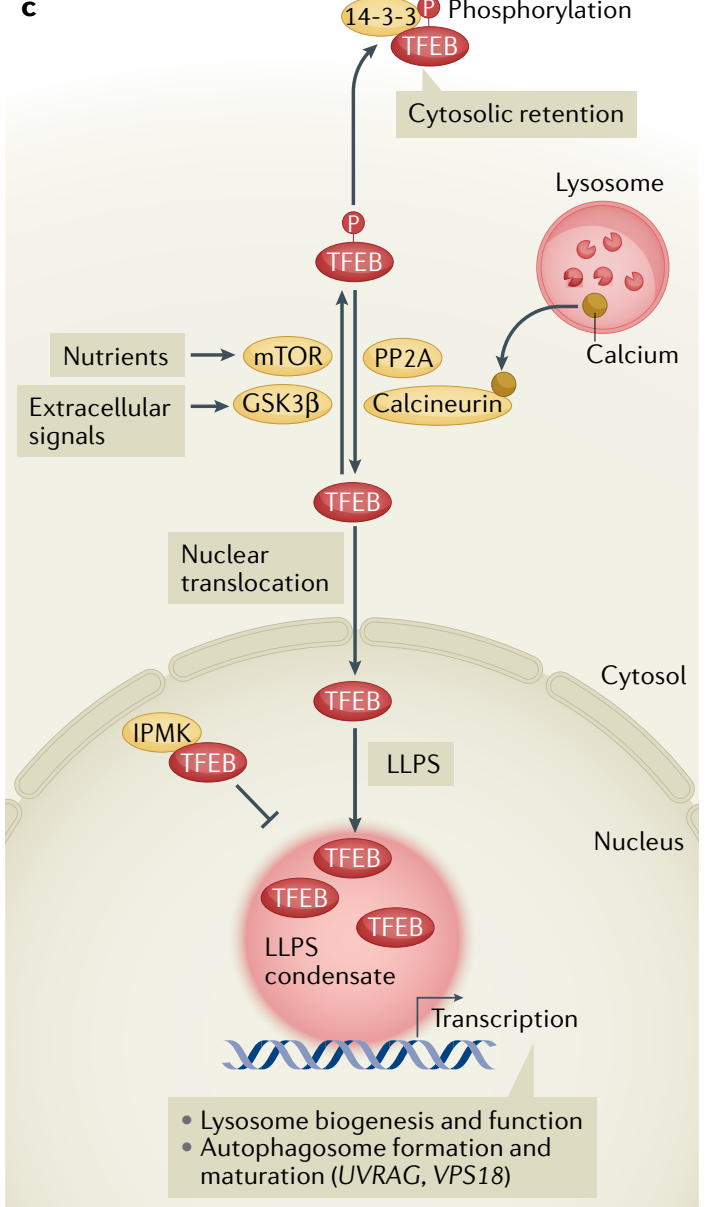

was rescued by promotion of RAB-7 dynamics on non-degradative autophagic vacuoles by expression of the GDP-bound form of RAB-7 or by promotion of lysosome biogenesis and function by depletion of the RBG-1-RBG-2 complex - a GEF and GAP complex known for promoting RAB protein dynamics for synaptic transmission and lipid droplet biogenesis. RBG-1RBG-2 has emerged as a new regulator of RAB-7 activity, which may act by targeting the RAB-7 GEF to lysosomes, or by promoting its activity on lysosomes ${ }^{101}$. These mechanisms, however, fail to suppress the autophagy defect caused by knockout of genes involved in autophagosome formation ${ }^{66,79,98,101}$.

Autophagosome maturation is intricately connected with endocytic trafficking pathways that are highly responsive to growth conditions. The heterogeneity and redundancy of the mechanisms governing autophagosome maturation confer flexibility and robustness on dynamic endocytic trafficking to maintain cellular homeostasis. 
4 Fig. 3 | Multiple mechanisms regulate autophagosome maturation. a|The SNARE domain of STX17 is modified by acetylation, a process controlled by the acetyltransferase CREBBP and the deacetylase HDAC2. Starvation inactivates CREBBP, resulting in deacetylation of STX17. Deacetylated STX17 interacts more strongly with the HOPS complex and SNAP29 and thus promotes autophagosome-lysosome fusion ${ }^{65}$. SNAP29 is O-GlcNAcylated by O-linked $\beta$ - $N$-acetylglucosamine (O-GlcNAc) transferase (OGT). This modification attenuates the assembly of SNAP29-containing SNARE complexes. Under starvation conditions that decrease the intracellular UDP-GlcNAc level, or in OGT-knockdown cells, the O-GlcNAcylation of SNAP29 is reduced, which in turn facilitates the assembly of the trans-SNARE complex for autophagosome maturation ${ }^{66}$. MTMR13, the guanine nucleotide exchange factor for the endosomal protein RAB21, controls RAB21-dependent trafficking of plasma membrane-localized VAMP8 to late endosomes/lysosomes ${ }^{63}$. Upon starvation, MTMR13 activates RAB21, which subsequently promotes the translocation of VAMP8 R SNARE to late endosomes/lysosomes to promote fusion of endosomes/lysosomes with autophagic vacuoles (see FIG. 1).b| Phosphatidylinositol 3-phosphate (Ptdlns3P) on autophagic vacuoles, generated by the UVRAG-c ontaining VPS34 complex, facilitates autophagosome maturation by recruiting the tethering factors HOPS complex. Rubicon interacts with the UVRAG-VPS34 complex and negatively regulates its function. Pacer is targeted by autophagic vacuole-localized SNARE STX17 and phosphoinositides (Ptdlns3P), and it antagonizes Rubicon and recruits the UVRAG-VPS34 complex to autophagic vacuoles. Pacer and UVRAG also recruit the HOPS complex. The RAB7 GTPase-activating protein Armus is targeted to autophagic vacuoles by interacting with LC3 and Ptdlns3P, and promotes RAB7 dynamics, whereby it is recycled from the autophagic vacuole membranes. This generates a mobile pool of RAB7 that can be recruited to endosomes to drive their maturation to late endosomes/ lysosomes. Depletion of the endoplasmic reticulum-localized transmembrane protein TMEM39A increases phosphatidylinositol 4-phosphate (Ptdlns4P) levels on late endosomes/ lysosomes (via inhibition of endoplasmic reticulum-to-Golgi apparatus trafficking of the Ptdlns4P phosphatase SAC1, not shown), probably by increasing Ptdlns4P levels in the trans-Golgi network (TGN), which promotes HOPS complex recruitment and enhances autophagosome-lysosome fusion. c| The transcription factor TFEB (as well as its homologue TFE3, not shown) activates the expression of genes involved in autophagy (including genes involved in autophagosome trafficking and fusion with lysosomes (UVRAG and VPS18)) and lysosomal biogenesis and function. The nuclear transport of TFEB is regulated by its phosphorylation levels. Various kinases, such as mTORC1 (downstream of nutrients) and glycogen synthase kinase $3 \beta$ (CSK3 $\beta$ ) (downstream of protein kinase $C$ (PKC) signalling in response to various extracellular signals), phosphorylate TFEB to prevent its nuclear import, while protein phosphatase 2A (PP2A) and calcineurin (which is activated by calcium release from lysosomes) dephosphorylate TFEB to facilitate its translocation to the nucleus. The activity of TFEB is also controlled by liquid-liquid phase separation (LLPS), whereby TFEB forms condensates that promote gene transcription. The nuclear protein inositol polyphosphate multikinase (IPMK) directly binds to TFEB and inhibits the formation of TFEB condensates.

\section{a-Synuclein \\ An aggregation-prone protein \\ that accumulates in Lewy \\ bodies and plays a central \\ role in the pathogenesis of \\ Parkinson disease and other \\ synucleinopathies.}

Polyglutamine expansion

An expansion of the CAG-

trinucleotide repeat in

disease-related genes resulting

in polyglutamine expansion

disorders such as Huntington

disease and spinocerebellar

ataxias.

X-linked spinal and bulbar muscular atrophy

An X-linked, recessive lower

motor neuron disease caused

by a CAG polyglutamine repeat

expansion within the first exon

of the androgen receptor gene.
Huntington disease, amyotrophic lateral sclerosis (ALS) and frontotemporal lobar dementia (FTLD). Notably, in these diseases, autophagosomes are formed but then accumulate as non-degradative autophagic vacuoles $^{102,103}$. Various mutations have been implicated in these neurodegenerative disorders (FIG. 4). Some of these mutations affect endocytic trafficking at the intersection with autophagosome maturation ${ }^{102}$. For example, mutations affecting the p150 subunit of the dynein-dynactin motor cause motor neuron disease ${ }^{104}$, while mutations in the ESCRT-III complex subunit CHMP2B cause familial ALS and FTLD ${ }^{105}$. Impaired lysosomal function is also related to the pathogenesis of neurodegenerative diseases. When lysosomal acidification is defective, fusion of lysosomes with autophagic vacuoles can still occur, but the resulting autolysosomes are non-degradative ${ }^{106}$. Mutant presenilin 1 , one of the major causes of familial Alzheimer disease, impairs lysosomal acidification ${ }^{107}$. Also, LSDs featuring dysfunctional, non-degradative autolysosomes, such as Gaucher disease, multiple sulfatase deficiency, mucopolysaccharidosis type IIIA and mucolipidosis type IV, are associated with neurodegeneration ${ }^{108,109}$. Impaired lysosomal biogenesis is also a feature of neurodegenerative disorders. In this case, disease proteins, such as accumulated $\alpha$-synuclein in Parkinson disease or androgen receptor with polyglutamine expansion in $X$-linked spinal and bulbar muscular atrophy sequester TFEB in the cytoplasm, impairing lysosomal function and autophagosome maturation ${ }^{110,111}$. Lysosomal function can also be impaired by physical damage instigated by disease proteins. Amyloid assemblies of diseaseassociated proteins, including $A \beta$ and tau in Alzheimer disease, $\alpha$-synuclein in Parkinson disease and huntingtin (HTT) with a pathological polyglutamine expansion in Huntington disease, can be secreted and transported between cells, and, following endocytosis, can induce endolysosome rupture in recipient cells ${ }^{112,113}$.

Mutations in genes directly involved in autophagosome maturation can also cause selective damage of certain populations of neurons, resulting in neurodegenerative features (FIG. 4). Mice deficient in Epg5 show selective loss of motor neurons and display key characteristics of $\mathrm{ALS}^{41}$. Recessive mutations in human EPG5 are associated with the multisystem disorder Vici syndrome ${ }^{114}$. Patients with Vici syndrome exhibit neurodevelopmental and neurodegenerative features that are recapitulated in Epg5-knockout mice ${ }^{115,116}$. Patients with Vici syndrome also display muscle abnormalities (skeletal muscle myopathy and cardiomyopathy $)^{115}$, which, as discussed later, have been linked to autophagosome maturation defects. In neural cells, WDR45 and WDR45B act redundantly during autophagosome maturation to target EPG5 to late endosomes/lysosomes ${ }^{25}$. De novo mutations in WDR45 cause $\beta$-propeller protein-associated neurodegeneration, previously known as static encephalopathy of childhood with neurodegeneration in adulthood ${ }^{117,118}$. Wdr45-knockout mice exhibit extensive swollen axons and impaired learning and memory, reminiscent of $\beta$-propeller protein-associated neurodegeneration ${ }^{119}$. A potential causative role of $W D R 45 B$ in intellectual disability recently emerged ${ }^{120}$. Wdr $45 b$-knockout mice 


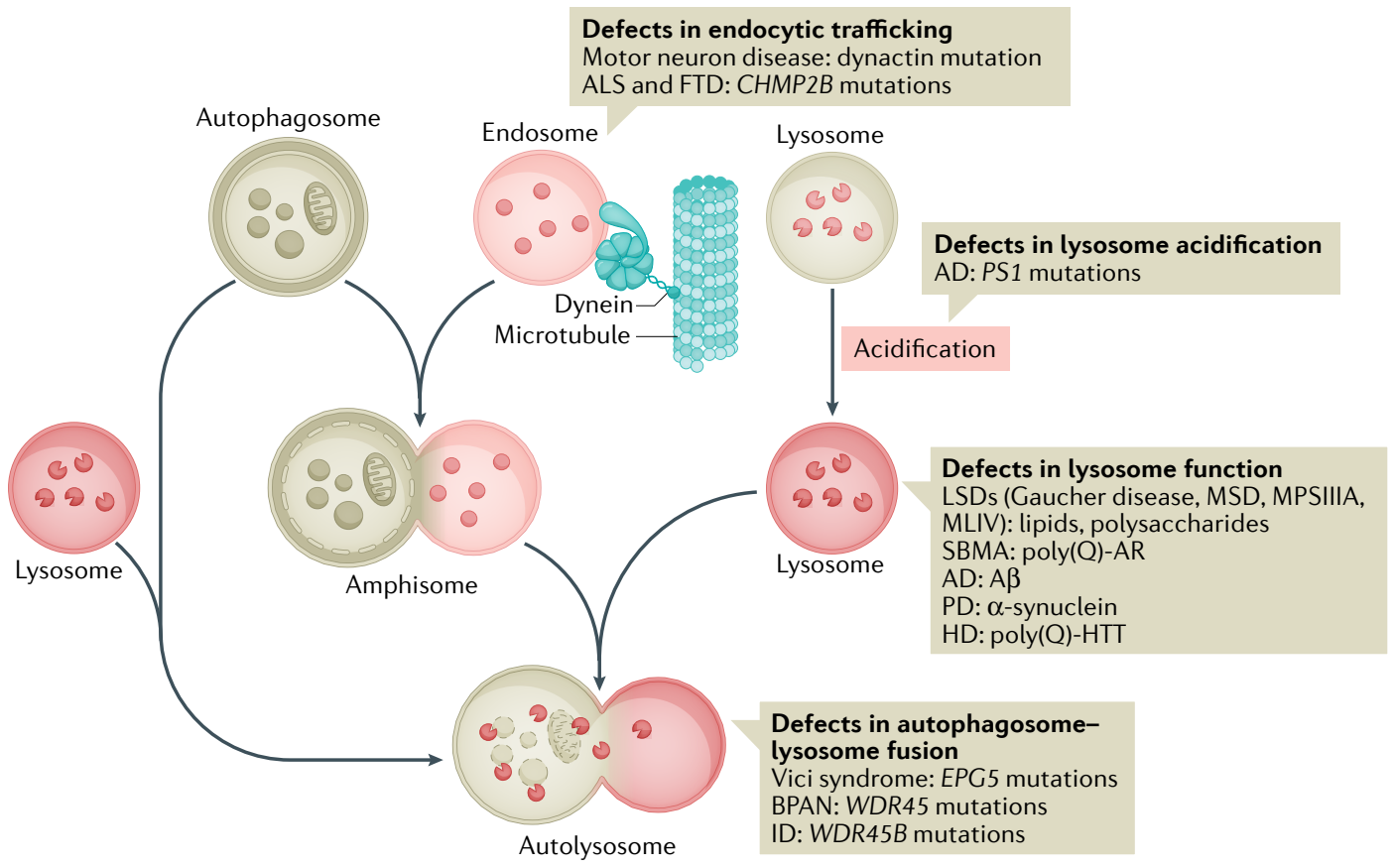

Fig. 4 | Deregulated autophagosome maturation in neurodegenerative diseases. Illustration of endolysosome trafficking and the progression of autophagosomes into autolysosomes. Impairments of several steps in the process have been linked to the pathogenesis of neurodegenerative diseases, and examples of the related diseases are shown. AD, Alzheimer disease; ALS, amyotrophic lateral sclerosis; BPAN, $\beta$-propeller protein-associated neurodegeneration; FTD, frontotemporal dementia; HD, Huntington disease; ID, intellectual disability; LSD, lysosomal storage disease; MLIV, mucolipidosis type IV; MPSIIIA, mucopolysaccharidosis type IIIA; MSD, multiple sulfatase deficiency; PD, Parkinson disease; poly(Q)-AR, androgen receptor with polyglutamine expansion; poly(Q)-HTT, huntingtin with polyglutamine expansion; SBMA, spinal and bulbar muscular atrophy.

A mixture of small peptides (37-43 amino acids) derived from sequential secretasemediated proteolytic processing of amyloid precursor protein (APP). $A \beta$ aggregates into insoluble neurotoxic plaques in patients with Alzheimer disease.

\section{Tau}

A major component of the

neurofibrillary tangles found in a number of neurodegenerative diseases, including Alzheimer disease.

\section{Vici syndrome}

A multisystem disorder (agenesis of the corpus callosum, progressive microcephaly, cataracts, hypopigmentation, combined immunodeficiency and recurrent infections, and cardiomyopathy) caused by mutations in the autophagy gene EPG5

\section{$\beta$-Propeller}

protein-associated neurodegeneration

A disease caused by WDR45 mutations. Patients show static psychomotor retardation in early childhood, then develop sudden-onset progressive parkinsonism, dystonia and cognitive impairment in early adulthood. also display abnormal motor behaviour and cognitive impairment, and pathologically exhibit cerebellar atrophy and accumulation of autophagosomes in swollen axons ${ }^{121}$.

Of note, mutations in different genes acting in autophagosome maturation result in distinct neuropathological deficits. This could be because the genes are differentially expressed in different neuronal subpopulations or are required in different types of neural cells or because their mutation results in accumulation of different types of autophagic cargo that may differ in different cells. The differential function of these genes in endocytic trafficking and recycling may also contribute to the distinct pathological defects.

Pathogenesis of muscle diseases. Autophagy is essential for preserving muscle mass and maintaining myofibre integrity ${ }^{122}$. Defects in lysosomal function and their degradative potential have been associated with a group of autophagic vacuolar myopathies, including Pompe disease, Danon disease and X-linked myopathy with excessive autophagy, which all present with accumulation of non-functional autophagic vacuoles ${ }^{123}$. Pompe disease is caused by deficiency of the lysosomal acid $\alpha$-glucosidase (GAA), which hydrolyses glycogen to glucose. It is characterized by massive accumulation of autophagic vacuoles, especially in skeletal muscles ${ }^{124}$. Patients with Danon disease, which is linked to deficiency in the lysosomal membrane protein LAMP2, display cardiomyopathy, myopathy and variable mental retardation ${ }^{125}$. Massive autophagic vacuoles accumulate in cardiomyocytes and skeletal muscle cells of patients with Danon disease and LAMP2-deficient mice ${ }^{125,126}$. $\mathrm{X}$-linked myopathy with excessive autophagy, a childhood-onset disease with progressive vacuolation and atrophy of skeletal muscle, is caused by compromised lysosomal acidification due to deficiency in the vacuolar ATPase assembly factor VMA21 (REF. ${ }^{127}$ ).

Muscle cells from patients with inclusion body myopathy, Paget disease of bone and frontotemporal dementia (IBMPFD)-related myopathy accumulate large, ubiquitin-positive rimmed vacuoles, which are non-digested autophagic vacuoles ${ }^{128}$. The causative gene for IBMPFD is VCP. VCP is primarily known for its roles in extraction of ubiquitylated proteins - which are often misfolded and prone to aggregation - from membranes or protein complexes for degradation or recycling. VCP knockdown or expression of IBMPFD mutant VCP results in accumulation of autophagic vacuoles containing ubiquitin-positive contents, and VCP was found to be essential for autophagosome maturation at late stages (following initial acidification), but the exact mechanisms through which VCP promotes further maturation are elusive ${ }^{128,129}$. VCP mutations are also associated with familial ALS ${ }^{103}$.

Deregulation in cancer. Autophagy functions as a tumour suppressor and promoter in a contextdependent manner ${ }^{130,131}$. Autophagy impedes tumour initiation and early stages of tumour progression by 
LAMP2

The receptor for

chaperone-mediated

autophagy. Mutations in

the gene encoding LAMP2

are causatively linked with

Danon disease.

EDEMosomes

Endoplasmic reticulum-derived

vesicles that tune endoplasmic

reticulum-associated

degradation activity by

targeting regulators of

endoplasmic reticulum-

associated degradation

(such as EDEM1) to lysosomes

for degradation.

ER-associated degradation (ERAD). A process involving

extraction of misfolded proteins from the endoplasmic reticulum (ER) for subsequent proteasomal degradation.

Secretion systems Apparatuses used by bacteria to secrete virulence factors from the bacterial cytosol into host cells or the host cell environment. acting in quality control processes such as removal of damaged mitochondria and maintenance of genomic stability. By contrast, in advanced tumours or during cancer therapy, autophagy enables tumour cells to survive harsh conditions such as hypoxia and metabolic stresses. In line with this, elevated lysosomal activity resulting from increased activity of TFEB and TFE3 which may be caused by chromosomal rearrangements, gene amplification or upregulation, and enhanced nuclear transport - is associated with the development or metastasis of various tumours, such as renal cell carcinomas, prostate cancer, pancreatic cancer, non-smallcell lung cancer (NSCLC) and breast cancer ${ }^{88,132}$. It is important to note that TFE3 and TFEB may also promote cancer progression, at least in part independently of their function in autophagy, by activating signalling pathways implicated in tumorigenesis, such as WNT and TGF $\beta$ signalling ${ }^{132}$. However, increased expression and nuclear import of TFE3 and TFEB have been demonstrated to maintain a high autophagy level to sustain intracellular amino acid pools in the pathogenesis of pancreatic ductal adenocarcinoma ${ }^{133}$. Enhanced TFEB activity can also promote lysosome exocytosis, releasing proteolytic enzymes, such as cathepsins, into the cell microenvironment, which fuels extracellular matrix remodelling, thereby stimulating cancer cell invasion and metastasis ${ }^{134}$.

Perturbation of autophagosome maturation machinery may also contribute to the occurrence and development of tumours. Alterations in EPG5 have been suggested to be involved in breast and prostate cancers $^{135,136}$. EPG5 expression is significantly lower in NSCLC clinical samples, and EPG5 knockdown promotes NSCLC cell proliferation and tumorigenesis ${ }^{137}$. WDR45 is genetically altered in patients with uterine corpus endometrial carcinoma and downregulated in cervical cancer development ${ }^{138,139}$. However, owing to the heterogeneity and redundancy in the autophagosome maturation machinery highlighted above, changes in individual components are probably not sufficient to drive tumorigenesis and tumorigenic progression, and it is likely that alterations in autophagosome maturation coexist with other oncogenic lesions, further aggravating pathology.

\section{Interplay with pathogen life cycles}

Autophagy responds to invading pathogens by capturing them and delivering them to lysosomes for degradation (a process known as xenophagy); this facilitates antigen presentation for activation of innate and adaptive immune responses ${ }^{15-17,140}$. Pathogens have evolved diverse strategies to inhibit autophagy at the initiation and/or maturation steps to escape destruction. Certain pathogens even block autophagosome maturation and subvert the resulting vesicles for their own benefit.

Harnessing of autophagosomes/amphisomes for viral replication and release. Positive-strand RNA viruses belonging to the family Picornaviridae, such as poliovirus, rhinovirus, coxsackievirus B3 and enterovirus D68, utilize double-membrane vesicles (DMVs) as membrane scaffolds for replication and transcription ${ }^{141-143}$.
DMVs formed in virus-infected cells are smaller than regular autophagosomes. They exhibit hallmarks of autophagosomes/amphisomes such as positivity for LC3 and the late endosomal/lysosomal marker LAMP1, but their further maturation into degradative autolysosomes is blocked ${ }^{14,144-146}$.

The betacoronaviruses, including mouse hepatitis virus (MHV), Middle East respiratory syndrome coronavirus, severe acute respiratory syndrome coronavirus (SARS-CoV) and SARS-CoV-2, also induce the formation of DMVs for anchoring the viral replication and transcription complexes ${ }^{32,147-149}$. The viral RNA products are localized in the DMV lumen and transported to the cytosol for translation and virion assembly via double-membrane-spanning molecular pores ${ }^{150}$. However, the canonical autophagic machinery, such as the LC3 lipidation system, is not required for DMV formation or coronavirus replication $^{151,152}$. Instead, the DMVs in MHV-infected cells are related to the ER-derived small vesicles, called 'EDEMosomes', that deliver short-lived regulators of ER-associated degradation (ERAD) to late endosomes/ lysosomes ${ }^{153}$. Certain autophagy proteins, however, are required for coronavirus infection. LC3 decorates the DMVs and is required for MHV replication ${ }^{153}$. Unlike on autophagic structures, where LC3 is conjugated with phosphatidylethanolamine, non-lipidated LC3 is present on the DMVs in MHV-infected cells ${ }^{153}$. Autophagy proteins involved in the generation of PtdIns3P are required for SARS-CoV-2 infection ${ }^{154,155}$. The ER-localized transmembrane autophagy proteins EPG3 (also known as VMP1) and TMEM41B are essential for autophagosome formation ${ }^{39,156-159}$. EPG3 and TMEM41B are also essential for replication of coronaviruses such as SARS-CoV-2 $\left(\operatorname{REFS}^{151,160}\right)$, but the step at which these proteins act during the viral life cycle has yet to be identified. In betacoronavirus-infected cells, the replicated viruses are transported inside lysosomes and released through the exocytic pathway ${ }^{161}$. Deacidification of lysosomes by loading with too many viral particles and/or by viral proteins such as ORF3a of SARS-CoV-2 promotes lysosomal exocytosis and thus viral egress from the infected cell ${ }^{32,161}$. Autophagosomes also can sequestrate and mediate non-lytic extracellular release of poliovirus, coxsackievirus B3 and enterovirus D68 (REFS ${ }^{141,145,162}$ ).

Bacterial survival and proliferation in autophagic vacuoles. Bacteria invade host cells via phagocytosis and reside in bacterium-containing vacuoles. If the vacuolar membrane is damaged, bacteria can escape into the cytosol. Autophagy captures bacteria in the cytosol or in damaged vacuoles and delivers them to lysosomes for destruction (via xenophagy) ${ }^{16,140}$. Bacteria use different secretion systems to deliver effectors or toxins to evade autophagy surveillance and even to exploit autophagic vacuoles for intracellular survival and growth. Bacterial virulence factors can block autophagy by inhibiting the autophagy induction signal, impairing autophagy recognition, or directly attenuating the function of autophagy proteins ${ }^{16,140}$. For example, the Legionella pneumophila effector protein RavZ inhibits host autophagy by functioning as 

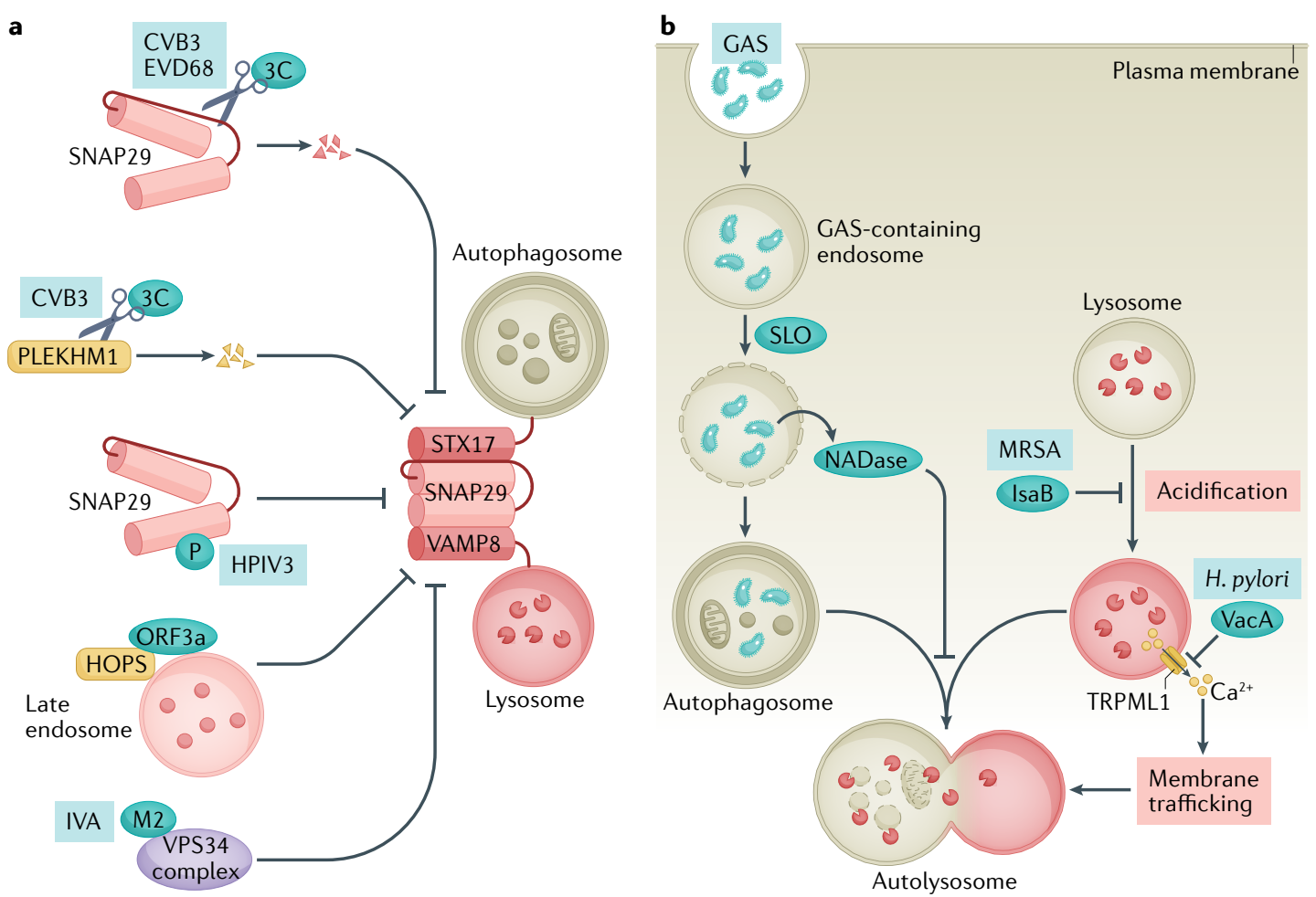

Fig. $5 \mid$ Mechanisms used by pathogens to interfere with autophagosome maturation. Autophagy can capture invading pathogens and deliver them to lysosomes for destruction. Pathogens (including viruses and bacteria) therefore use various mechanisms to block autophagosome-lysosome fusion to escape autophagy clearance. Pathogens can also modulate autophagic structures - including induction of accumulation of autophagic vacuoles or autophagy-independent doublemembrane vesicles marked by certain autophagosomal proteins and modulation of endolysosomal degradative enzymes and $\mathrm{pH}$ - to promote their own survival, replication and/or release (not shown). a / Viral proteinase 3C of coxsackievirus B3 (CVB3) and enterovirus D68 (EVD68) cleaves SNAP29 to reduce SNARE assembly ${ }^{14,145}$. Proteinase 3C of CVB3 also cleaves the tether protein PLEKHM1. Phosphoprotein (P) of human parainfluenza virus type 3 (HPIV3) competes with STX17 for SNAP29 binding ${ }^{176}$. ORF3a of severe acute respiratory syndrome coronavirus 2 (SARS-CoV-2) sequestrates the HOPS complex on late endosomes, thus impairing SNARE complex assembly. M2 protein of influenza virus A (IVA) dampens the activity of the VPS34 complex to prevent autophagosome maturation. $\mathbf{b}$ | Streptolysin O (SLO) damages the membrane of group A Streptococcus (GAS)-containing endosomes to trigger their engulfment by autophagosomes. Translocation of the co-toxin NAD-glycohydrolase (NADase) into the cytoplasm blocks the fusion of GAS-containing autophagic vacuoles with lysosomes ${ }^{213}$. The virulence factor IsaB of methicillin-resistant Staphylococcus aureus (MRSA) blocks lysosomal acidification to suppress the function of autolysosomes ${ }^{214}$. The virulence factor VacA of Helicobacter pylori prevents TRPML1-mediated calcium efflux from endosomes to disrupt endolysosomal trafficking and thus autophagosome maturation ${ }^{215}$.

a cysteine protease that uncouples lipid-conjugated ATG8 proteins ${ }^{163}$. RavZ cleaves ATG8 proteins between the carboxy-terminal glycine and the penultimate aromatic residue, producing ATG8 proteins that cannot be reconjugated ${ }^{163}$. SopF, the effector of Salmonella enterica subsp. enterica serovar Typhimurium, impairs initiation of xenophagy by interfering with the binding of the $\mathrm{V}$-ATPase on damaged bacterium-containing vacuoles to ATG16L $\mathrm{L}^{164}$. Maturation of bacterium-containing autophagic vacuoles (autophagosomes or fused vesicles of bacterium-containing vacuoles and autophagosomes) into degradative autolysosomes by deposition of lytic enzymes can also be inhibited. In macrophages, autophagic vacuoles containing the virulent strain of Mycobacterium tuberculosis (H37Rv) fail to recruit RAB7 for maturation into autolysosomes ${ }^{165,166}$. Vacuoles containing Mycobacterium marinum and Yersinia pestis exhibit features of non-degradative autolysosomes that are devoid of lysosomal enzymes ${ }^{167,168}$. Some bacteria (for example, Serratia marcescens, Staphylococcus aureus, Anaplasma phagocytophilum and Coxiella burnetii) even exploit autophagic vacuoles as replication niches for intracellular growth and proliferation ${ }^{169-174}$. Fusion with autophagosomes facilitates the formation of vacuoles where bacteria reside and replicate - by promoting fusion of individual bacterium-containing vacuoles and/or providing a membrane for vacuole expansion and also supplies nutrients to the pathogen ${ }^{171,172,174,175}$. In line with this, replication of these pathogens is promoted by autophagy induction and is blocked by autophagy inhibition $^{172-174}$.

Mechanisms used by pathogens to interfere with autophagosome maturation. Inhibition of the assembly of STX17-SNAP29-VAMP8 is widely used by viruses to prevent the formation of degradative autolysosomes and/or to accumulate autophagosomes/amphisomes for their replication or release (FIG. 5a). Viral proteinase 
Tat-beclin 1

A cell-penetrating peptide

that induces autophagy.

Eighteen amino acids from

the evolutionarily conserved

domain of beclin 1 are

covalently coupled with the

HIV-1 Tat protein transduction

domain.

Lysosomotropic agents

Substances that are taken

up selectively into lysosomes

irrespective of their chemical

nature or uptake mechanism

Aptamers

Short, single-stranded DNA or RNA molecules or peptides

that non-covalently bind to a target molecule with high

affinity and specificity.

Nanobodies

Antibodies consisting of

a single monomeric heavy

chain variable domain used

in pharmaceutical applications

such as diagnosis and therapy

due to their small size,

solubility and stability.

Chloroquine

(CQ). An antimalarial drug

also commonly used as a

lysosomotropic agent that

changes the lysosomal $\mathrm{pH}$
3C of coxsackievirus B3 and enterovirus D68 mediates cleavage of SNAP29, separating the two SNARE motifs and thus impairing the formation of the SNARE complex $^{144,145}$. The phosphoprotein (P) of human parainfluenza virus type 3 binds to SNAP29 to inhibit its interaction with STX17 (REF. ${ }^{176}$ ), whereas cells in which hepatitis $\mathrm{C}$ virus is replicating exhibit reduced levels of STX17 due to its decreased expression and increased turnover ${ }^{177}$. Tethering factors can also be targeted by viral effectors. PLEKHM1 is proteolytically targeted by proteinase $3 \mathrm{C}$ of coxsackievirus $\mathrm{B} 3$ to separate the HOPS complex-binding and LC3-binding amino terminus from the RAB7-interacting carboxy terminus, thus abolishing its tethering function ${ }^{145}$ (FIG. 5a). Cells infected with SARS-CoV-2, or expressing the viral accessory protein ORF3a, sequester components of the HOPS complex on late endosomes ${ }^{32}$. This prevents functional HOPS complex from interacting with STX17 and consequently inhibits assembly of the STX17SNAP29-VAMP8 complex ${ }^{32}$ (FIG. 5a). M2 protein of influenza virus A inhibits autophagosome maturation by interfering with the beclin 1-containing and UVRAGcontaining VPS34 complex ${ }^{178}$ (FIG. 5a). Bacterial virulence factors also block the maturation and elimination of bacterium-containing autophagic vacuoles, but the mechanisms are less well understood. Examples are shown in FIG. 5b.

\section{Therapeutic interventions}

Different steps of the autophagy pathway are potential targets for therapeutic interventions ${ }^{179,180}$. Inhibitors and activators of autophagosome formation, including VPS34 and ULK1 inhibitors or the activating peptide Tat-beclin 1, are potent modulators of autophagy, but are not yet available for clinical use ${ }^{181,182}$. By contrast, lysosomotropic agents, which inhibit the activity of lysosomes and block their fusion with autophagic vesicles, have been used in several clinical trials ${ }^{183-185}$. Modulation of autophagosome maturation through targeting key components of the maturation machinery or through controlling autolysosomal activity by targeting the transcriptional programme of lysosome and autophagosome biogenesis is also a potential therapeutic option to regulate autophagic flux, but such approaches require further optimization if they are to be used in a clinical setting. Inhibiting and stimulating autophagosome maturation are important therapeutic avenues to explore. In many neurodegenerative diseases, autophagosome maturation is blocked ${ }^{186,187}$, so restoring the autophagy flux would be an important therapeutic option. When inhibition of autophagy is required, as, for example, in cancer cells, it is important to consider the step at which the autophagic pathway must be interrupted. Different outcomes can be observed when autophagy is blocked at autophagosome formation or autophagosome maturation. In line with this, it has been shown that blocking maturation of autophagosomes favours cell death via necroptosis in human cancer cells in response to TNF-related apoptosis-inducing ligand (TRAIL), whereas blocking the formation of autophagosomes triggers TRAIL-dependent apoptosis in the same cells ${ }^{188}$.
Targeting the autophagosome maturation machinery. Targeting the machinery that controls autophagosome maturation (such as ESCRT, SNAREs and the HOPS complex) or targeting post-translational modifications of these proteins would be ideal to either increase or decrease autophagy flux ${ }^{189-191}$. Inhibitors of $O$-GlcNAc transferase and those of the opposing enzyme, $O$-GlcNAcase, can stimulate or inhibit autophagosome maturation, respectively, by modulating the O-GlcNAcylation of SNAP29 (REFS ${ }^{66,192,193}$ ) (TABLE 1). As many cellular proteins are $O$-GlcNAcylated, blocking $O$-GlcNAc sites on a specific protein would require the development of methods to increase the selectivity of targeting, such as the use of aptamers or nanobodies ${ }^{194}$. Another strategy to inhibit autophagosome maturation would be to use the small molecule TCH-165 to specifically activate the proteasomal degradation of SNAP29 and STX17 (REF. ${ }^{195}$ ). Inhibiting Rubicon is a potential approach to promote autophagy; however, pharmacological Rubicon inhibitors have yet to be developed. This strategy must be carefully evaluated because Rubicon is a positive modulator of LC3-associated phagocytosis, a process known to have a protective effect in many inflammatory diseases ${ }^{196-198}$.

Modulation of the transcription programme of autophagosome maturation. Modulation of TFEB/TFE3 is an obvious strategy to control autophagy on a transcriptional level. It has been shown that TFEB overexpression has beneficial effects in ameliorating LSDs and obesity by stimulating lipophagy ${ }^{199-201}$. However, chronic overexpression of TFEB favours the progression of pancreatic tumours and NSCLCs ${ }^{200}$. Thus, developing small molecules to acutely stimulate TFEB and harness the autophagy-lysosomal pathway would be beneficial in ameliorating diseases in which autophagy has a defensive role ${ }^{201}$. Small molecules can activate TFEB indirectly by modulating its upstream kinases or phosphatases (TABLE 1). For example, TFEB nuclear transport is promoted by rapamycin via inhibiting mTORC1 activity or by compounds isolated from the herb Euphorbia peplus Linn via the PKC-GSK $3 \beta$ cascade $^{90}$.

Blocking autophagosome maturation with lysosomotropic agents. Chloroquine (CQ), hydroxychloroquine (HCQ) and their derivatives are the only clinically approved drugs that act on autophagosome maturation (TABLE 1). They are used alone or in combination with other drugs, mostly in ongoing oncology trials, in general with the goal of optimizing therapies by blocking autophagy induced by cancer treatments ${ }^{183-185}$. The newgeneration dimeric CQ derivatives Ly05 and DQ661 are active at lower concentrations than CQ and $\mathrm{HCQ}^{130,183,202}$.

CQ and HCQ block autophagy flux by inhibiting the hydrolytic capacity of autolysosomes. They increase the $\mathrm{pH}$ in autolysosomal compartments and hence block the activity of acidic proteases and other enzymes ${ }^{203}$. Beyond their capacity for $\mathrm{H}^{+}$trapping, monomeric and dimeric CQ derivatives also block lysosomal function by inhibiting the activity of the lysosomal enzyme palmitoyl-protein thioesterase 1 (PPT1), which is involved in stabilizing the lysosomal localization of 
Table 1 | Different strategies used to modulate autophagosome maturation

\begin{tabular}{|c|c|c|}
\hline Function on autophagy & Potential therapeutic strategy & Pharmacological mechanism \\
\hline \multirow[t]{4}{*}{ Autophagy activator } & Inhibitors of $O$-GlcNAc transferase & $\begin{array}{l}\text { Stimulating autophagy by suppressing the } \\
\text { O-GlcNAcylation of SNAP29 }\end{array}$ \\
\hline & Small molecules to stimulate TFEB activity & $\begin{array}{l}\text { Promoting transcription of the autophagy- } \\
\text { lysosome pathway genes and enhancing } \\
\text { lysosomal biogenesis }\end{array}$ \\
\hline & mTOR or GSK $3 \beta$ inhibitors & $\begin{array}{l}\text { Suppressing TFEB phosphorylation to } \\
\text { promote TFEB nuclear translocation }\end{array}$ \\
\hline & $\begin{array}{l}\text { Small molecules to block Rubicon } \\
\text { interaction with the VPS34-UVRAG } \\
\text { complex }\end{array}$ & $\begin{array}{l}\text { Stimulating autophagy by activating the } \\
\text { VPS34-UVRAG complex }\end{array}$ \\
\hline \multirow[t]{6}{*}{ Autophagy inhibitor } & Inhibitors of $O$-GlcNAcase & $\begin{array}{l}\text { Inhibiting autophagosome maturation by } \\
\text { facilitating the O-GlcNAcylation of SNAP } 29\end{array}$ \\
\hline & $\begin{array}{l}\text { Chloroquine, hydroxychloroquine and } \\
\text { their derivatives }\end{array}$ & Inhibiting autolysosomal hydrolysis \\
\hline & Inhibitors of V-ATPase & $\begin{array}{l}\text { Blocking the transport of protons for } \\
\text { lysosomal acidification }\end{array}$ \\
\hline & Inhibitors of lysosomal hydrolases & Blocking degradation by lysosomes \\
\hline & $\mathrm{TCH}-165$ & $\begin{array}{l}\text { Inhibiting autophagosome fusion with } \\
\text { lysosomes by activating proteasomal } \\
\text { degradation of STX17 and SNAP29 }\end{array}$ \\
\hline & PIKFYVE kinase inhibitors & $\begin{array}{l}\text { Inhibiting lysosome fission and causing } \\
\text { enlarged non-functional lysosomes }\end{array}$ \\
\hline
\end{tabular}

GSK3 $\beta$, glycogen synthase kinase $3 \beta$; O-GlcNAc, O-linked $\beta$ - $N$-acetylglucosamine.

V-ATPase subunits ${ }^{202}$. Thus, inhibition of PPT1 results in autophagy inhibition. In addition, lysosomal function and/or fusion with autophagosomes could be targeted by other means of $\mathrm{pH}$ regulation (such as via modulation of V-ATPase), inhibition of the cation channel TRPML1, inhibition of lysosomal enzymes or modulation of lysosomal membrane dynamics - fusion and fission - which impact on lysosomal number and function ${ }^{179,180,183,204}$ (TABLE 1).

Of note, lysosomotropic agents target all acidic compartments and also other pathways ${ }^{203,205}$, and thus in some cases the beneficial effects of lysosomotropic agents can be attributed to mechanisms other than a blockade of autophagy ${ }^{130,203}$. For example, these drugs inhibit tumour progression, independently of the autophagy blockade, by altering the trafficking of signalling molecules (that is, NOTCH1) in the endocytic pathway ${ }^{206}$ and by other mechanisms ${ }^{184,203}$. It is also worth mentioning that the activity of CQ and HCQ observed in vitro may not be the same in vivo due to different parameters. For example, during viral infection, the cell type used in vitro may not reflect the tropism of the virus in vivo and/or the sensitivity to $\mathrm{CQ}^{207}$. Moreover, the acidic environment in tumours can protonate the lysosomotropic agent and greatly reduce its cellular uptake ${ }^{208}$.

\section{Conclusions and perspectives}

Autophagosome maturation is an essential step in the autophagy pathway that ensures the formation of degradative autolysosomes. It adds another layer of complexity and provides an extra node to integrate nutrient status and stresses for regulation of autophagic degradation. The distinct organization and trafficking of the endolysosomal compartment in different cell types and growth conditions add complexity at the intersection of the autophagy and endocytic pathways. Thus, the trans-SNARE complexes and tethering factors act coordinately with context-specific factors to mediate fusion of autophagosomes with endocytic vesicles and lysosomes. Further investigations are needed to elucidate how different signalling pathways and stresses coordinate autophagosome initiation and maturation to ensure efficient progression of autophagic flux and how these processes are adapted in different cell types or pathophysiological contexts.

Autophagosome maturation is widely manipulated by pathogens to escape from destruction and for replication and growth. Pathogens that use autophagic vacuoles for replication can both activate autophagosome initiation and block maturation to achieve their maximal accumulation. Understanding how viral proteins and bacterial virulence factors modulate host autophagy will help us to develop strategies to interfere with the pathogen-host interaction and even to restore autophagy as a defence mechanism. Such strategies are urgently required with the evolution of multidrug-resistant bacteria. Elucidating the underlying mechanisms for autophagosome maturation defects and deregulation of the function of the autophagosome-lysosome system is also key for us to understand the pathogenesis of various human diseases. Targeting autophagosome maturation - via modulation of SNAREs, tethers and their regulators as well as lysosome biogenesis and function - offers an effective strategy for the treatment of these diseases.

Biomarkers and methods that reliably monitor autophagy flux in vivo are needed to examine temporal changes of autophagy activity and to evaluate interventions that target autophagosome maturation. 
Positron emission

tomography

An imaging technique that

visualizes metabolic changes

by three-dimensional

mapping of administered

positron-emitting

radiopharmaceuticals
A combination of assays has been used to measure autophagy flux and to monitor autophagosome maturation ${ }^{25}$. However, many of these assays are difficult to implement in humans. Several methods have recently been developed to serve as reliable autophagy biomarkers in humans ${ }^{209}$. Analysis of autophagy flux in isolated peripheral blood mononuclear cells is used to measure autophagy activity in human blood samples ${ }^{210,211}$. Positron emission tomography can be used with hypoxia tracers to correlate hypoxia and autophagy in tumours and also to gauge the level of specific autophagy substrates in tissues by the use of positron emission tomography ligands that bind to autophagy substrates ${ }^{184,209}$.
The levels of specific molecules in biological fluids can also be used to determine autophagy flux in tissues. For instance, the blood level of arginase 1 reflects autophagy activity in the liver ${ }^{212}$. Nevertheless, these methods are of low throughout and/or can be applied only to selected cells or tissues. Thus, to screen drugs targeting autophagy, there is an urgent need for reliable, high-throughput clinical biomarkers to measure autophagic activity by the identification of tissue-specific circulating autophagy by-products and the development of flux probes for use in imaging techniques.

Published online 23 July 2021
1. Feng, Y. C., He, D., Yao, Z. Y. \& Klionsky, D. J. The machinery of macroautophagy. Cell Res. 24, 24-41 (2014).

2. Lamb, C. A., Yoshimori, T. \& Tooze, S. A. The autophagosome: origins unknown, biogenesis complex. Nat. Rev. Mol. Cell Biol. 14, 759-774 (2013).

3. Nakatogawa, H. Mechanisms governing autophagosome biogenesis. Nat. Rev. Mol. Cell. Biol. 21, 439-458 (2020).

4. Mizushima, N., Yoshimori, T. $\&$ Ohsumi, Y. The role of Atg proteins in autophagosome formation. Annu. Rev. Cell Dev. Biol. 27, 107-132 (2011).

5. Stolz, A., Ernst, A. \& Dikic, I. Cargo recognition and trafficking in selective autophagy. Nat. Cell Biol. 16, 495-501 (2014).

6. Takahashi, Y. et al. An autophagy assay reveals the ESCRT-III component CHMP2A as a regulator of phagophore closure. Nat. Commun. 9, 2855 (2018).

7. Takahashi, Y et al. VPS37A directs ESCRT recruitment for phagophore closure. J. Cell Biol. 218, 3336-3354 (2019).

8. Zhen, Y. et al. ESCRT-mediated phagophore sealing during mitophagy. Autophagy 16, 826-841 (2020)

9. Ktistakis, N. T. \& Tooze, S. A. Digesting the expanding mechanisms of autophagy. Trends Cell Biol. 26 624-635 (2016)

10. Zhao, Y. G. \& Zhang, H. Formation and maturation of autophagosomes in higher eukaryotes: a social network. Curr. Opin. Cell Biol. 53, 29-36 (2018).

11. Zhao, Y. G. \& Zhang, H. Autophagosome maturation: an epic journey from the ER to lysosomes. J. Cell Biol. 218, 757-770 (2019).

12. $\mathrm{Pu}$, J., Guardia, C. M., Keren-Kaplan, T. \& Bonifacino, J. S. Mechanisms and functions of lysosome positioning. J. Cell Sci. 129, 4329-4339 (2016).

13. Jiang, P. D. \& Mizushima, N. Autophagy and human diseases. Cell Res. 24, 69-79 (2014).

14. Choi, Y., Bowman, J. W. \& Jung, J. U. Autophagy during viral infection - a double-edged sword. Nat. Rev. Microbiol. 16, 341-354 (2018).

15. Deretic, V., Saitoh, T. \& Akira, S. Autophagy in infection, inflammation and immunity. Nat. Rev. Immunol. 13, 722-737 (2013).

16. Kimmey, J. M. \& Stallings, C. L. Bacterial pathogens versus autophagy: implications for therapeutic interventions. Trends Mol. Med. 22, 1060-1076 (2016).

17. Levine, B., Mizushima, N. \& Virgin, H. W. Autophagy in immunity and inflammation. Nature 469, 323-335 (2011).

18. Filimonenko, M. et al. Functional multivesicular bodies are required for autophagic clearance of protein aggregates associated with neurodegenerative disease. J Cell Biol 179, 485-500 (2007).

19. Razi, M., Chan, E. Y. W. \& Tooze, S. A. Early endosomes and endosomal coatomer are required for autophagy. J. Cell Biol. 185, 305-321 (2009).

20. Jahn, R. \& Scheller, R. H. SNAREs - engines for membrane fusion. Nat. Rev. Mol. Cell Biol. 7 631-643 (2006)

21. Langemeyer, L., Frohlich, F. \& Ungermann, C. Rab GTPase function in endosome and lysosome biogenesis. Trends Cell Biol. 28, 957-970 (2018).

22. Yu, I. M. \& Hughson, F. M. Tethering factors as organizers of intracellular vesicular traffic. Annu. Rev. Cell Dev. Biol. 26, 137-156 (2010).

23. Nguyen, T. N. et al. Atg8 family LC3/GABARAP proteins are crucial for autophagosome-lysosome fusion but not autophagosome formation during PINK 1/Parkin mitophagy and starvation. J. Cell Biol. 215, 857-874 (2016).

24. Vaites, L. P., Paulo, J. A., Huttlin, E. L. \& Harper, J. W. Systematic analysis of human cells lacking ATG8 proteins uncovers roles for GABARAPs and the CCZ1/ MON1 regulator C18orf8/RMC1 in macroautophagic and selective autophagic flux. Mol. Cell Biol. 38, e00392-17 (2018)

25. Ji, C. C., Zhao, H. Y., Chen, D., Zhang, H. \& Zhao, Y. C $\beta$-Propeller proteins WDR45 and WDR45B control autophagosome maturation into autolysosomes in neural cells. Curr. Biol. 31, 1666-1677.e6 (2021).

26. Itakura, E., Kishi-Itakura, C. \& Mizushima, N. The hairpin-type tail-anchored SNARE syntaxin 17 targets to autophagosomes for fusion with endosomes/ lysosomes. Cell 151, 1256-1269 (2012). This study reveals that the SNARE complex composed of autophagosomal Qa SNARE STX17, Obc SNARE SNAP29 and Qc SNARE VAMP8 mediates autophagosome-late endosome/ lysosome fusion.

27. Matsui, T. et al. Autophagosomal YKT6 is required for fusion with lysosomes independently of syntaxin 17 J. Cell Biol. 217, 2633-2645 (2018).

28. Takats, S. et al. Autophagosomal syntaxin 17-dependent lysosomal degradation maintains neuronal function in Drosophila. J. Cell Biol. 201 531-539 (2013)

29. Tsuboyama, K. et al. The ATG conjugation systems are important for degradation of the inner autophagosomal membrane. Science 354, 1036-1041 (2016).

30. Kumar, S. et al. Mechanism of Stx 17 recruitment to autophagosomes via IRGM and mammalian Atg8 proteins. J. Cell Biol. 217, 997-1013 (2018)

31. Diao, J. J. et al. ATG 14 promotes membrane tethering and fusion of autophagosomes to endolysosomes. Nature 520, 563-566 (2015)

32. Miao, G. et al. ORF3a of the COVID-19 virus SARS CoV-2 blocks HOPS complex-mediated assembly of the SNARE complex required for autolysosome formation. Dev. Cell 56, 427-442.e425 (2021). In this work, the authors demonstrate that the late endosome/lysosome-localized ORF3a protein of SARS-CoV-2 prevents fusion of autophagic vacuoles with lysosomes by sequestrating the HOPS complex component VPS39 and hence blocking the assembly of the SNARE complex required for the formation of degradative autolysosomes.

33. Takats, S. et al. Interaction of the HOPS complex with syntaxin 17 mediates autophagosome clearance in Drosophila. Mol. Biol. Cell 25, 1338-1354 (2014).

34. Jiang, P. et al. The HOPS complex mediates autophagosome-lysosome fusion through interaction with syntaxin 17. Mol. Biol. Cell 25, 1327-1337 (2014).

35. Stroupe, C., Collins, K. M., Fratti, R. A. \& Wickner, W. Purification of active HOPS complex reveals its affinities for phosphoinositides and the SNARE Vam7p. EMBO J. 25, 1579-1589 (2006)

36. Gao, J. Q., Langemeyer, L., Kummel, D., Reggiori, F. \& Ungermann, C. Molecular mechanism to target the endosomal Mon 1-Ccz1 GEF complex to the pre-autophagosomal structure. eLife 7, e31145 (2018).

37. Hegedus, K. et al. The Ccz1-Mon 1-Rab7 module and Rab5 control distinct steps of autophagy. Mol. Biol. Cell 27, 3132-3142 (2016).
38. Manil-Segalen, M. et al. The C. elegans LC3 acts downstream of GABARAP to degrade autophagosomes by interacting with the HOPS subunit VPS39. Dev, Cell 30, 110-110 (2014).

39. Tian, Y. et al. C. elegans screen identifies autophagy genes specific to multicellular organisms. Cell 141 , 1042-1055 (2010).

40. Wang, Z. et al. The Vici syndrome protein EPG5 is a Rab7 effector that determines the fusion specificity of autophagosomes with late endosomes/lysosomes. Mol. Cell 63, 781-795 (2016).

This study shows that EPG5, the gene causing Vici syndrome, encodes a tether protein that is recruited to late endosomes/lysosomes by binding to RAB7 and facilitates SNARE complex formation to mediate autophagosome maturation.

41. Zhao, H. Y. et al. Mice deficient in Epg5 exhibit selective neuronal vulnerability to degeneration. J. Cell Biol. 200, 731-741 (2013).

42. McEwan, D. G. et al. PLEKHM1 regulates autophagosome-lysosome fusion through HOPS complex and LC3/GABARAP proteins. Mol. Cell 57 39-54 (2015)

The authors demonstrate that PLEKHM1 tethers autophagosomes and lysosomes by simultaneously binding to autophagosomal LC3 and lysosome-localized RAB7, and also promotes autophagosome-lysosome fusion by recruiting the HOPS complex.

43. Wijdeven, R. H. et al. Cholesterol and ORP1Lmediated ER contact sites control autophagosome transport and fusion with the endocytic pathway. Nat. Commun. 7, 11808 (2016).

44. Wetzel, L. et al. TECPR 1 promotes aggrephagy by direct recruitment of LC3C autophagosomes to lysosomes. Nat. Commun. 11, 2993 (2020).

45. Chen, D. D. et al. A mammalian autophagosome maturation mechanism mediated by TECPR 1 and the Atg12-Atg5 conjugate. Mol. Cell 45, 629-641 (2012).

46. Jahreiss, L. Menzies, F. M. \& Rubinsztein, D. C The itinerary of autophagosomes: from peripheral formation to kiss-and-run fusion with lysosomes. Traffic 9, 574-587 (2008).

47. Korolchuk, V. I. et al. Lysosomal positioning coordinates cellular nutrient responses. Nat Cell Biol. 13, 453-460 (2011)

48. Bonifacino, J. S. \& Neefjes, J. Moving and positioning the endolysosomal system. Curr. Opin. Cell Biol. 47, 1-8 (2017).

49. Jia, R. Guardia, C. M., Pu, J., Chen, Y \& Bonifacino, J. S. BORC coordinates encounter and fusion of lysosomes with autophagosomes. Autophagy 13, 1648-1663 (2017).

50. Pankiv, S. et al. FYCO1 is a Rab7 effector that binds to LC3 and PI3P to mediate microtubule plus end-directed vesicle transport. J. Cell Biol. 188, 253-269 (2010).

51. Johansson, M. et al. Activation of endosomal dynein motors by stepwise assembly of Rab7-RILPp150Glued, ORP1L, and the receptor betalll spectrin J. Cell Biol. 176, 459-471 (2007).

52. Rocha, N. et al. Cholesterol sensor ORP $1 \mathrm{~L}$ contacts the ER protein VAP to control Rab7-RILP-p150(Glued) and late endosome positioning. J. Cell Biol. 185 1209-1225 (2009).

53. van der Kant, R. et al. Late endosomal transport and tethering are coupled processes controlled by RILP and the cholesterol sensor ORP1 L. J. Cell Sci. 126 3462-3474 (2013). 
54. Lee, S., Sato, Y. \& Nixon, R. A. Lysosomal proteolysis inhibition selectively disrupts axonal transport of degradative organelles and causes an Alzheimer's-like axonal dystrophy. J. Neurosci. 31, 7817-7830 (2011).

55. Maday, S., Wallace, K. E. \& Holzbaur, E. L. F. Autophagosomes initiate distally and mature during transport toward the cell soma in primary neurons. J. Cell Biol. 196, 407-417 (2012).

This work reveals that in primary neurons, autophagosomes formed at the distal tip of the axon undergo retrograde transport to the cell soma, accompanied by gradual maturation and acidification

56. Fu, M. M., Nirschl, J. J. \& Holzbaur, E. L. F. LC3 binding to the scaffolding protein JIP regulates processive dynein-driven transport of autophagosomes. Dev. Cell 29, 577-590 (2014).

57. Cason, S. E. et al. Sequential dynein effectors regulate axonal autophagosome motility in a maturationdependent pathway. Preprint at bioRxiv https://doi. org/10.1101/2020.11.01.363333 (2020).

58. Russell, R. C., Yuan, H. X. \& Guan, K. L. Autophagy regulation by nutrient signaling. Cell Res. 24, 42-57 (2014).

59. Furuya, T. et al. Negative regulation of Vps34 by Cdk mediated phosphorylation. Mol. Cell 38, 500-511 (2010).

60. Nazio, F. et al. mTOR inhibits autophagy by controlling ULK1 ubiquitylation, self-association and function through AMBRA1 and TRAF6. Nat. Cell Biol. 15, 406-416 (2013)

61. Nazio, F. et al. Fine-tuning of ULK1 mRNA and protein levels is required for autophagy oscillation. J. Cell Biol. 215, 841-856 (2016).

62. Liu, C. C. et al. Cul3-KLHL20 ubiquitin ligase governs the turnover of ULK1 and VPS34 complexes to control autophagy termination. Mol. Cell 61, 84-97 (2016).

63. Jean, S., Cox, S., Nassari, S. \& Kiger, A. A. Starvationinduced MTMR 13 and RAB2 1 activity regulates VAMP8 to promote autophagosome-lysosome fusion. EMBO Rep. 16, 297-311 (2015).

64. Fraldi, A. et al. Lysosomal fusion and SNARE function are impaired by cholesterol accumulation in lysosomal storage disorders. EMBO J. 29, 3607-3620 (2010).

65. Shen, Q. H. et al. Acetylation of STX17 (syntaxin 17) controls autophagosome maturation. Autophagy 17 1157-1169 (2021)

66. Guo, B. et al. O-GIcNAc-modification of SNAP-29 regulates autophagosome maturation. Nat. Cell Biol. 16, 1215-1226 (2014)

67. Hanover, J. A., Krause, M. W. \& Love, D. C. The hexosamine signaling pathway: O-GIcNAc cycling in feast or famine. Biochim. Biophys. Acta 1800, 80-95 (2010).

68. Mizushima, N. A brief history of autophagy from cell biology to physiology and disease. Nat. Cell Biol. 20 , 521-527 (2018)

69. Funderburk, S. F., Wang, Q. J. \& Yue, Z. Y. The beclin 1 -VPS34 complex - at the crossroads of autophag and beyond. Trends Cell Biol. 20, 355-362 (2010).

70. Matsunaga, K. et al. Two beclin 1-binding proteins, Atg $14 \mathrm{~L}$ and Rubicon, reciprocally regulate autophagy at different stages. Nat. Cell Biol. 11, 385-396 (2009).

71. Cheng, X. W. et al. Pacer mediates the function of class III PI3K and HOPS complexes in autophagosome maturation by engaging Stx 17. Mol. Cell 65 1029-1043 (2017)

72. Liang, C. Y. et al. Beclin 1-binding UVRAG targets the class C Vps complex to coordinate autophagosome maturation and endocytic trafficking. Nat. Cell Biol. 10, 776-787 (2008)

73. Sun, Q. M., Westphal, W., Wong, K. N., Tan, I. $\&$ Zhong, Q. Rubicon controls endosome maturation as a Rab7 effector. Proc. Natl Acad. Sci. USA 107, 19338-19343 (2010).

74. Zhong, Y. et al. Distinct regulation of autophagic activity by Atg $14 \mathrm{~L}$ and Rubicon associated with beclin 1-phosphatidylinositol-3-kinase complex. Nat. Cell Biol. 11, 468-476 (2009).

75. Kim, Y. M. et al. mTORC1 phosphorylates UVRAG to negatively regulate autophagosome and endosome maturation. Mol. Cell 57, 207-218 (2015)

76. Judith, D. et al. ATC9A shapes the forming autophagosome through arfaptin 2 and phosphatidylinositol 4-kinase III beta. J. Cell Biol. 218, 1634-1652 (2019).

77. Wang, H. Z. et al. GABARAPs regulate PI4Pdependent autophagosome: lysosome fusion Proc. Natl Acad. Sci. USA 112, 7015-7020 (2015).
78. Del Bel, L. M. \& Brill, J. A. Sac1, a lipid phosphatase at the interface of vesicular and nonvesicular transport. Traffic 19, 301-318 (2018).

79. Miao, G., Zhang, Y., Chen, D. \& Zhang, H. The ERlocalized transmembrane protein TMEM39A/SUSR2 regulates autophagy by controlling the trafficking of the Ptdlns(4)P phosphatase SAC1. Mol. Cell 77 , 618-632.e5 (2020).

80. Jaber, N. et al. Vps34 regulates Rab7 and late endocytic trafficking through recruitment of the GTPase-activating protein Armus. J. Cell Sci. 129 4424-4435 (2016)

81. Carroll, B. et al. The TBC/RabGAP armus coordinates Rac1 and Rab7 functions during autophagy. Dev. Cell 25, 15-28 (2013)

82. Piper, R. C. \& Luzio, J. P. Late endosomes: sorting and partitioning in multivesicular bodies. Traffic $\mathbf{2}$. 612-621 (2001)

83. Baba, T., Toth, D. J., Sengupta, N., Kim, Y. J. \& Balla, T. Phosphatidylinositol 4,5-bisphosphate controls Rab7 and PLEKHM1 membrane cycling during autophagosome-lysosome fusion. EMBO J. 38 e100312 (2019)

84. Raben, N. \& Puertollano, R. TFEB and TFE3: linking lysosomes to cellular adaptation to stress. Annu. Rev. Cell Dev. Biol. 32, 255-278 (2016)

85. Sardiello, M. et al. A gene network regulating lysosomal biogenesis and function. Science 325 , 473-477 (2009)

86. Settembre, C. et al. TFEB links autophagy to lysosomal biogenesis. Science 332, 1429-1433 (2011).

87. Ballabio, A. \& Bonifacino, J. S. Lysosomes as dynamic regulators of cell and organismal homeostasis. Nat. Rev. Mol. Cell Biol. 21, 101-118 (2020).

88. Puertollano, R., Ferguson, S. M., Brugarolas, J. \& Ballabio, A. The complex relationship between TFEB transcription factor phosphorylation and subcellular localization. EMBO J. 37, e98804 (2018).

89. Settembre, C. et al. A lysosome-to-nucleus signalling mechanism senses and regulates the lysosome via mTOR and TFEB. EMBO J. 31, 1095-1108 (2012).

90. Li, Y. et al. Protein kinase $C$ controls lysosome biogenesis independently of mTORC1. Nat. Cell Biol. 18, 1065-1077 (2016)

91. Medina, D. L. et al. Lysosomal calcium signalling regulates autophagy through calcineurin and TFEB. Nat. Cell Biol. 17, 288-299 (2015).

This study shows that upon activation by lysosomal calcium release through MCOLN1, the phosphatase calcineurin dephosphorylates TFEB and promotes its nuclear translocation to induce autophagy and lysosomal biogenesis.

92. Martina, J. A. \& Puertollano, R. Protein phosphatase 2A stimulates activation of TFEB and TFE3 transcription factors in response to oxidative stress. J. Biol. Chem 293, 12525-12534 (2018)

93. Banani, S. F., Lee, H. O., Hyman, A. A. \& Rosen, M. K Biomolecular condensates: organizers of cellular biochemistry. Nat. Rev. Mol. Cell Biol. 18, 285-298 (2017).

94. Shin, Y. \& Brangwynne, C. P. Liquid phase condensation in cell physiology and disease. Science 357, eaaf4382 (2017).

95. Zhang, H. et al. Liquid-liquid phase separation in biology: mechanisms, physiological functions and human diseases. Sci. China Life Sci 63, 953-985 (2020).

96. Boija, A. et al. Transcription factors activate genes through the phase-separation capacity of their activation domains. Cell 175, 1842-1855 (2018).

97. Sabari, B. R. Biomolecular condensates and gene activation in development and disease. Dev. Cell 55 , 84-96 (2020).

98. Chen, D. et al. Inositol polyphosphate multikinase inhibits liquid-liquid phase separation of TFEB to negatively regulate autophagy activity. $\mathrm{Dev}$. Cell 55 588-602 (2020).

This study demonstrates that the TFEB condensate formed via LLPS is involved in transcription and its formation is negatively regulated by the nuclear-localized protein IPMK

99. Tabata, K. et al. Rubicon and PLEKHM1 negatively regulate the endocytic/autophagic pathway via a novel Rab7-binding domain. Mol. Biol. Cell. 21 4162-4172 (2010).

100. Fujita, N. et al. Genetic screen in Drosophila muscle identifies autophagy-mediated T-tubule remodeling and a Rab2 role in autophagy. eLife 6, e23367 (2017)

101. Wang, Z. Y. et al. The RBG-1-RBG-2 complex modulates autophagy activity by regulating lysosomal biogenesis and function in C. elegans. J. Cell Sci. 132 jcs234195 (2019).
102. Nixon, R. A., Yang, D. S. \& Lee, J. H. Neurodegenerative lysosomal disorders: a continuum from development to late age. Autophagy 4, 590-599 (2008).

103. Otomo, A., Pan, L. \& Hadano, S. Dysregulation of the autophagy-endolysosomal system in amyotrophic lateral sclerosis and related motor neuron diseases. Neurol. Res. Int. 2012, 498428 (2012).

104. Puls, I. et al. Mutant dynactin in motor neuron disease. Nat. Genet. 33, 455-456 (2003).

105. Parkinson, N. et al. ALS phenotypes with mutations in CHMP2B (charged multivesicular body protein 2B) Neurology $67,1074-1077$ (2006).

106. Mauvezin, C., Nagy, P., Juhasz, G. ¿ Neufeld, T. P. Autophagosome-lysosome fusion is independent of V-ATPase-mediated acidification. Nat. Commun. $\mathbf{6}$, 7007 (2015).

107. McBrayer, M \& Nixon, R. A Lysosome and calcium dysregulation in Alzheimer's disease: partners in crime. Biochem. Soc. Trans. 41, 1495-1502 (2013).

108. Settembre, C. et al. A block of autophagy in lysosomal storage disorders. Hum. Mol. Genet. 17, 119-129 (2008).

109. Vergarajauregui, S., Connelly, P. S., Daniels, M. P. $\&$ Puertollano, R. Autophagic dysfunction in mucolipidosis type IV patients. Hum. Mol. Genet. 17, 2723-2737 (2008).

110. Decressac, M. et al. TFEB-mediated autophagy rescues midbrain dopamine neurons from alphasynuclein toxicity. Proc. Natl Acad. Sci. USA 110, E1817-E1826 (2013).

111. Cortes, C. J. et al. Polyglutamine-expanded androgen receptor interferes with TFEB to elicit autophagy defects in SBMA. Nat. Neurosci. 17, 1180-1189 (2014).

This work reveals that the polyglutamine-expanded androgen receptor physically binds to and inactivates TFEB, thereby impairing autophagy function and contributing to the pathogenesis of spinal and bulbar muscular atrophy.

112. Flavin, W. P. et al. Endocytic vesicle rupture is a conserved mechanism of cellular invasion by amyloid proteins. Acta. Neuropathol. 134, 629-653 (2017).

113. Jiang, P. Z., Gan, M., Yen, S. H., McLean, P. J. \& Dickson, D. W. Impaired endo-lysosomal membrane integrity accelerates the seeding progression of alpha-synuclein aggregates. Sci. Rep. 7, 28794446 (2017).

114. Cullup, T. et al. Recessive mutations in EPG5 cause Vici syndrome, a multisystem disorder with defective autophagy. Nat. Genet. 45, 83-87 (2013).

115. Byrne, S., Dionisi-Vici, C., Smith, L., Gautel, M. \& Jungbluth, H. Vici syndrome: a review. Orphanet $J$. Rare Dis. 11, 21 (2016)

116. Zhao, Y. G., Zhao, H. Y., Sun, H. Y. \& Zhang, H. Role of Epg5 in selective neurodegeneration and Vici syndrome. Autophagy 9, 1258-1262 (2013).

117. Haack, T. B. et al. Exome sequencing reveals de novo WDR45 mutations causing a phenotypically distinct, X-linked dominant form of NBIA. Am. J. Hum. Genet. 91, 1144-1149 (2012)

118. Saitsu, H. et al. De novo mutations in the autophagy gene WDR45 cause static encephalopathy of childhood with neurodegeneration in adulthood. Nat. Genet. 45, 445-449 (2013).

119. Zhao, Y. G. et al. The autophagy gene Wdr45/Wipi4 regulates learning and memory function and axonal homeostasis. Autophagy 11, 881-890 (2015).

120. Suleiman, J. et al. WDR45B-related intellectual disability, spastic quadriplegia, epilepsy, and cerebral hypoplasia: a consistent neurodevelopmental syndrome. Clin. Genet. 93, 360-364 (2018).

121. Ji, C. C. et al. Role of Wdr45b in maintaining neural autophagy and cognitive function. Autophagy 16 615-625 (2020)

122. Jiao, J. Q. \& Demontis, F. Skeletal muscle autophagy and its role in sarcopenia and organismal aging. Curr. Opin. Pharmacol. 34, 1-6 (2017).

123. Dowling, J. J., Moore, S. A., Kalimo, H. \& Minassian, B. A. X-linked myopathy with excessive autophagy: a failure of self-eating. Acta. Neuropathol. 129, 383-390 (2015)

124. Myerowitz, R., Puertollano, R. \& Raben, N. Impaired autophagy: the collateral damage of lysosomal storage disorders. EBioMedicine 63 103166 (2021).

125. Nishino, I. et al. Primary LAMP-2 deficiency causes X-linked vacuolar cardiomyopathy and myopathy (Danon disease). Am. J. Hum. Genet. 67, 390-390 (2000).

126. Tanaka, Y. et al. Accumulation of autophagic vacuoles and cardiomyopathy in LAMP-2-deficient mice. Nature 406, 902-906 (2000) 
127. Saraste, A. et al. No cardiomyopathy in X-linked myopathy with excessive autophagy. Neuromuscul. Disord. 25, 485-487 (2015)

128. Tresse, E. et al. VCP/p97 is essential for maturation of ubiquitin-containing autophagosomes and this function is impaired by mutations that cause IBMPFD. Autophagy 6, 217-227 (2010).

129. Ju, J. S. et al. Valosin-containing protein (VCP) is required for autophagy and is disrupted in VCP disease. J. Cell Biol. 187, 875-888 (2009).

130. Towers, C. G., Wodetzki, D. \& Thorburn, A Autophagy and cancer: modulation of cell death pathways and cancer cell adaptations. J. Cell Biol. 219, e201909033 (2020)

131. Dikic, I. \& Elazar, Z. Mechanism and medical implications of mammalian autophagy. Nat. Rev. Mol. Cell Biol. 19, 349-364 (2018).

132. Astanina, E., Bussolino, F. \& Doronzo, G. Multifaceted activities of transcription factor EB in cancer onset and progression. Mol. Oncol. 15, 327-346 (2021).

33. Perera, R. M. et al. Transcriptional control of autophagy-lysosome function drives pancreatic cancer metabolism. Nature 524, 361-365 (2015). The authors demonstrate that pancreatic ductal adenocarcinoma cells contain higher levels of nuclear MITF, TFE3 and TFEB than normal cells, which in turn activate autophagy to promote tumour maligancy.

134. Kundu, S. T. et al. TMEM $106 \mathrm{~B}$ drives lung cancer metastasis by inducing TFEB-dependent lysosome synthesis and secretion of cathepsins. Nat. Commun. 9, 2731 (2018)

135. Sjoblom, T. et al. The consensus coding sequences of human breast and colorectal cancers. Science 314, 268-274 (2006)

136. Wu, B. et al. Intratumoral heterogeneity and genetic characteristics of prostate cancer. Int. J. Cancer 146. 3369-3378 (2020)

137. Li, H. et al. C-myc/miR-150/EPG5 axis mediated dysfunction of autophagy promotes development of non-small cell lung cancer. Theranostics 9 . 5134-5148 (2019)

138. Bai, M. X., Che, Y. Y., Lu, K. \& Fu, L. Analysis of deubiquitinase OTUD5 as a biomarker and therapeutic target for cervical cancer by bioinformatic analysis. PeerJ 8, e9146 (2020).

139. Lebovitz, C. B. et al. Cross-cancer profiling of molecular alterations within the human autophagy interaction network. Autophagy 11, 1668-1687 (2015).

140. Huang, J. \& Brumell, J. H. Bacteria-autophagy interplay: a battle for survival. Nat. Rev. Microbiol. 12, 101-114 (2014).

141. Jackson, W. T. et al. Subversion of cellular autophagosomal machinery by RNA viruses. PLoS Biol. 3, 861-871 (2005)

142. Wong, H. H. \& Sanyal, S. Manipulation of autophagy by (+) RNA viruses. Semin. Cell Dev. Biol. 101, 3-11 (2020).

143. Wong, J. et al. Autophagosome supports coxsackievirus B3 replication in host cells. J. Virol. 82, 9143-9153 (2008)

144. Corona, A. K., Saulsbery, H. M., Velazquez, A. F. C. \& Jackson, W. T. Enteroviruses remodel autophagic trafficking through regulation of host SNARE proteins to promote virus replication and cell exit. Cell Rep. 22 3304-3314 (2018)

145. Mohamud, Y. et al. Enteroviral infection inhibits autophagic flux via disruption of the SNARE complex to enhance viral replication. Cell Rep. 22, 3292-3303 (2018).

146. Kemball, C. C. et al. Coxsackievirus infection induces autophagy-like vesicles and megaphagosomes in pancreatic acinar cells in vivo. J. Virol. 84 12110-12124 (2010)

147. Knoops, K. et al. SARS-coronavirus replication is supported by a reticulovesicular network of modified endoplasmic reticulum. PLoS Biol. 6, 1957-1974 (2008).

148. Miller, K. et al. Coronavirus interactions with the cellular autophagy machinery. Autophagy 16 2131-2139 (2020)

149. Snijder, E. J. et al. A unifying structural and functional model of the coronavirus replication organelle: tracking down RNA synthesis. PLoS Biol. 18, e3000715 (2020).

150. Wolff, G. et al. A molecular pore spans the double membrane of the coronavirus replication organelle. Science 369, 1395-1398 (2020).

151. Schneider, W. M. et al. Genome-scale identification of SARS-CoV-2 and pan-coronavirus host factor networks. Cell 184, 120-132.e14 (2021).
152. Zhao, Z. J. et al. Coronavirus replication does not require the autophagy gene ATC5. Autophagy 3 , 581-585 (2007)

153. Reggiori, F. et al. Coronaviruses hijack the LC3-Ipositive EDEMosomes, ER-derived vesicles exporting short-lived ERAD regulators, for replication. Cell Host Microbe 7, 500-508 (2010).

Using MHV infection as a model, the authors show that coronaviruses hijack LC3-labelled EDEMsomes as DMVs for their replication

154. Dniloski, Z. et al. Identification of required host factors for SARS-CoV-2 infection in human cells. Cell 184 92-105.e16 (2021)

155. Wang, R. et al. Genetic screens identify host factors for SARS-CoV-2 and common cold coronaviruses. Cell 184, 106-119.e14 (2021).

156. Morita, K. et al. Genome-wide CRISPR screen identifies TMEM41B as a gene required for autophagosome formation. J. Cell Biol. 217 3817-3828 (2018)

157. Zhao, Y. G. et al. The ER-localized transmembrane protein EPG-3/VMP1 regulates SERCA activity to control ER-isolation membrane contacts for autophagosome formation. Mol. Cell 67, 974-989 (2017).

158. Moretti, F. et al. TMEM41B is a novel regulator of autophagy and lipid mobilization. EMBO Rep. 19, e45889 (2018)

159. Shoemaker, C. J. et al. CRISPR screening using an expanded toolkit of autophagy reporters identifies TMEM 41B as a novel autophagy factor. PLoS Biol. 17 e2007044 (2019).

160. Hoffmann, H. H. et al. TMEM41B is a pan-flavivirus host factor. Cell 184, 133-148 (2021).

161 . Ghosh, S. et al. $\beta$-Coronaviruses use lysosomes for egress instead of the biosynthetic secretory pathway. Cell 183, 1520-1535 (2020).

This is the first demonstration that betacoronaviruses exploit lysosomal exocytosis for egress, accompanied by blockage of lysosomal acidification, inactivation of lysosomal degradation and impaired antigen presentation.

162. Bird, S. W., Maynard, N. D., Covert, M. W. \& Kirkegaard, K. Nonlytic viral spread enhanced by autophagy components. Proc. Natl Acad. Sci. USA 111, 13081-13086 (2014).

163. Choy, A et al. The Legionella effector RavZ inhibits host autophagy through irreversible Atg8 deconjugation. Science 338, 1072-1076 (2012)

164. Xu, Y. et al. A bacterial effector reveals the VATPase-ATG 16L1 axis that initiates xenophagy. Cell 178, 552-566 (2019).

This study demonstrates that the Salmonella Typhimurium T3SS effector SopF blocks V-ATPase on damaged bacterium-containing vacuoles from recruiting ATG16L1 to initiate xenophagy.

165. Chandra, P. et al. Mycobacterium tuberculosis inhibits RAB7 recruitment to selectively modulate autophagy flux in macrophages. Sci. Rep. 5, 16320 (2015).

166. Romagnoli, A. et al. ESX-1 dependent impairment of autophagic flux by Mycobacterium tuberculosis in human dendritic cells. Autophagy 8, 1357-1370 (2012).

167. Lerena, M. C. \& Colombo, M. I. Mycobacterium marinum induces a marked LC3 recruitment to its containing phagosome that depends on a functional ESX-1 secretion system. Cell Microbiol. 13, 814-835 (2011).

168. Pujol, C. et al. Yersinia pestis can reside in autophagosomes and avoid xenophagy in murine macrophages by preventing vacuole acidification. Infect. Immun 77, 2251-2261 (2009).

169. Beron, W., Gutierrez, M. G., Rabinovitch, M. \& Colombo, M. I. Coxiella burnetii localizes in a Rab7. labeled compartment with autophagic characteristics. Infect. Immun. 70, 5816-5821 (2002).

170. Fedrigo, G. V., Campoy, E. M., Di Venanzio, G., Colombo, M. I. \& Vescovi, E. G. Serratia marcescen is able to survive and proliferate in autophagic-like vacuoles inside non-phagocytic cells. PLOS ONE 6 e24054 (2011)

171. Niu, H., Xiong, Q. M., Yamamoto, A., Hayashi-Nishino, M. \& Rikihisa, Y. Autophagosomes induced by a bacterial beclin 1 binding protein facilitate obligatory intracellular infection. Proc. Natl Acad. Sci. USA 109, 20800-20807 (2012).

172. Niu, H., Yamaguchi, M. \& Rikihisa, Y. Subversion of cellular autophagy by Anaplasma phagocytophilum. Cell Microbiol. 10, 593-605 (2008).

173. Schnaith, A. et al. Staphylococcus aureus subver autophagy for induction of caspase-independent host cell death. J. Biol. Chem. 282, 2695-2706 (2007).
174. Winchell, C. G., Graham, J. G., Kurten, R. C. \& Voth D. E. Coxiella burnetii type IV secretion-dependent recruitment of macrophage autophagosomes. Infect. Immun. 82, 2229-2238 (2014).

175. Newton, H. J. et al. A screen of Coxiella burnetii mutants reveals important roles for Dot/lcm effectors and host autophagy in vacuole biogenesis. PLoS Pathog. 10, e1004286 (2014).

176. Ding, B. B. et al. Phosphoprotein of human parainfluenza virus type 3 blocks autophagosomelysosome fusion to increase virus production Cell Host Microbe 15, 564-577 (2014). This study demonstrates that the viral phosphoprotein $(P)$ of human parainfluenza virus type 3 interacts with SNAP29 to prevent its binding to STX17, thereby blocking fusion of autophagosomes with lysosomes to avoid autophagic degradation.

177. Ren, H. et al. The autophagosomal SNARE protein syntaxin 17 is an essential factor for the hepatitis $C$ virus life cycle. J. Virol. 90, 5989-6000 (2016).

178. Gannage, M. et al. Matrix protein 2 of influenza A virus blocks autophagosome fusion with lysosomes. Cell Host Microbe 6, 367-380 (2009).

179. Galluzzi, L., Bravo-San Pedro, J. M., Levine, B., Green, D. R. \& Kroemer, G. Pharmacological modulation of autophagy: therapeutic potential and persisting obstacles. Nat. Rev. Drug Discov. 16 487-511 (2017)

180. Morel, E. et al. Autophagy: a druggable process. Annu. Rev. Pharmacol. Toxicol. 57, 375-398 (2017).

181. Levine, B., Packer, M. \& Codogno, P. Development of autophagy inducers in clinical medicine. J. Clin. Invest. 125, 14-24 (2015).

182. Vakifahmetoglu-Norberg, H., Xia, H. G. \& Yuan, J. Pharmacologic agents targeting autophagy. J. Clin. Invest. 125, 5-13 (2015).

183. Amaravadi, R. K., Kimmelman, A. C. \& Debnath, J. Targeting autophagy in cancer: recent advances and future directions. Cancer Discov. 9, 1167-1181 (2019).

184. Levy, J. M. M., Towers, C. G. \& Thorburn, A. Targeting autophagy in cancer. Nat. Rev. Cancer 17, 528-542 (2017).

185. Marsh, T., Tolani, B. \& Debnath, J. The pleiotropic functions of autophagy in metastasis. J. Cell Sci. 134 jcs247056 (2021).

186. Djajadikerta, A. et al. Autophagy induction as a therapeutic strategy for neurodegenerative diseases. J. Mol. Biol. 432, 2799-2821 (2020).

187. Ganesan, D. \& Cai, Q. Understanding amphisomes. Biochem. J. 478, 1959-1976 (2021)

188. Goodall, M. L. et al. The autophagy machinery controls cell death switching between apoptosis and necroptosis. Dev. Cell 37, 337-349 (2016).

189. Corona, A. K. \& Jackson, W. T. Finding the middle ground for autophagic fusion requirements. Trends Cell Biol. 28, 869-881 (2018).

190. van der Beek, J., Jonker, C., van der Welle, R., Liv, N. \& Klumperman, J. CORVET, CHEVI and HOPS multisubunit tethers of the endo-lysosomal system in health and disease. J. Cell Sci. 132, jcs 189134 (2019).

191. Vietri, M., Radulovic, M. \& Stenmark, H. The many functions of ESCRTs. Nat. Rev. Mol. Cell Biol. 21, 25-42 (2020)

192. Dodson, M. et al. Increased O-GlcNAcylation of SNAP29 drives arsenic-induced autophagic dysfunction. Mol. Cell Biol. 38, e00595-17 (2018).

193. Zhou, F. X. et al. Down-regulation of OGT promotes cisplatin resistance by inducing autophagy in ovarian cancer. Theranostics 8, 5200-5212 (2018).

194. Zhu, Y. \& Hart, G. W. Targeting O-GIcNAcylation to develop novel therapeutics. Mol. Aspects Med. 79, 100885 (2020).

195. Njomen, E. \& Tepe, J. J. Regulation of autophagic flux by the $20 \mathrm{~S}$ proteasome. Cell Chem. Biol. 26 , 1283-1294.e5 (2019).

196. Galluzzi, L. \& Green, D. R. Autophagy-independent functions of the autophagy machinery. Cell 177 , 1682-1699 (2019).

197. Wan, J. H. et al. LC3-associated phagocytosis protects against inflammation and liver fibrosis via immunoreceptor inhibitory signaling. Sci. Transl. Med. 12, eaaw8523 (2020).

198. Wong, S. W., Sil, P. \& Martinez, J. Rubicon: LC3associated phagocytosis and beyond. FEBS J. 285 , 1379-1388 (2018).

199. Settembre, C et al. TFEB controls cellular lipid metabolism through a starvation-induced autoregulatory loop. Nat. Cell Biol. 15, 1016-1016 (2013). 
200. Napolitano, G. \& Ballabio, A. TFEB at a glance. J. Cell Sci. 129, 2475-2481 (2016).

201. Parenti, G., Medina, D. L. \& Ballabio, A. The rapidly evolving view of lysosomal storage diseases. EMBO Mol. Med. 13, e12836 (2021).

202. Rebecca, V. W. et al. PPT1 promotes tumor growth and is the molecular target of chloroquine derivatives in cancer. Cancer Discov. 9, 220-229 (2019).

203. Nirk, E. L., Reggiori, F. \& Mauthe, M. Hydroxychloroquine in rheumatic autoimmune disorders and beyond. EMBO Mol. Med. 12, e12476 (2020).

204. Whitmarsh-Everiss, T. \& Laraia, L. Small molecule probes for targeting autophagy. Nat. Chem. Biol. 17 653-664 (2021)

205. Chude, C. I. \& Amaravadi, R. K. Targeting autophagy in cancer: update on clinical trials and novel inhibitors. Int. J. Mol. Sci. 18, 1279 (2017).

206. Maes, H. et al. Tumor vessel normalization by chloroquine independent of autophagy. Cancer Cell 26, 190-206 (2014).

207. Hoffmann, M. et al. Chloroquine does not inhibit infection of human lung cells with SARS-CoV-2. Nature 585, 588-590 (2020).

208. Pellegrini, P. et al. Acidic extracellular pH neutralizes the autophagy-inhibiting activity of chloroquine: implications for cancer therapies. Autophagy 10, 562-571 (2014)

209. Mizushima, N. \& Murphy, L. O. Autophagy assays for biological discovery and therapeutic development. Trends Biochem. Sci. 45, 1080-1093 (2020).

210. Pietrocola, F. et al. Metabolic effects of fasting on human and mouse blood in vivo. Autophagy 13 , 567-578 (2017)

211. Bensalem, J. et al. Measurement of autophagic flux in humans: an optimized method for blood samples. Autophagy https://doi.org/10.1080/15548627.2020. 1846302 (2020)

212. Poillet-Perez, L. et al. Autophagy maintains tumour growth through circulating arginine. Nature 565 . 569-573 (2018).
213. O'Seaghdha, M. \& Wessels, M. R. Streptolysin O and its co-toxin NAD-glycohydrolase protect group A streptococcus from xenophagic killing. PLoS Pathog. 9, e1003394 (2013).

The authors show that during group A

Streptococcus infection, the pore-forming toxin streptolysin $\mathrm{O}$ damages the bacterium-containing vacuole, resulting in release of active NADase into the cytosol, which prevents the fusion of group A Streptococcus-containing autophagosomes with lysosomes.

214. Liu, P. F et al. IsaB inhibits autophagic flux to promote host transmission of methicillin-resistant Staphylococcus aureus. J. Invest. Dermatol. 135 2714-2722 (2015).

215. Capurro, M. I. et al. VacA generates a protective intracellular reservoir for Helicobacter pylori that is eliminated by activation of the lysosomal calcium channel TRPML1. Nat. Microbiol. 4, 1411-1423 (2019).

216. Zhang, H. Lipid transfer at ER-isolation membrane contacts. Nat Rev. Mol Cell Biol. 21, 121 (2020).

217. Schutter, M., Giavalisco, P., Brodesser, S. \& Graef, M. Local fatty acid channeling into phospholipid synthesis drives phagophore expansion during autophagy. Cell 180, 135-149.e14 (2020).

218. Yang C \& Wang X Lysosome biogenesis: regulation and functions. J. Cell Biol. 220, e202102001 (2021).

219. Teter, S. A. et al. Degradation of lipid vesicles in the yeast vacuole requires function of $\mathrm{Cvt} 17$, a putative lipase. J. Biol. Chem. 276, 2083-2087 (2001).

220. Liu, Y. B. et al. Autophagy-dependent ribosomal RNA degradation is essential for maintaining nucleotide homeostasis during C. elegans development. eLife 7, e36588 (2018).

221. Liu, B., Du, H. W., Rutkowski, R., Gartner, A. \& Wang, X. C. LAAT-1 is the lysosomal lysine/arginine transporter that maintains amino acid homeostasis. Science 337, 351-354 (2012).

222. Rong, Y. G. et al. Spinster is required for autophagic Iysosome reformation and $\mathrm{mTOR}$ reactivation following starvation. Proc. Natl Acad. Sci. USA 108 7826-7831 (2011).
223. Yu, L. et al. Termination of autophagy and reformation of lysosomes regulated by mTOR. Nature 465 , 942-946 (2010)

224. Chen, Y. \& Yu, L. Development of research into autophagic lysosome reformation. Mol. Cell 41 45-49 (2018).

225. Sun, T., Wang, X. W., Lu, Q., Ren, H. Y. \& Zhang, H. CUP-5, the $C$. elegans ortholog of the mammalian lysosomal channel protein MLN1/TRPML1, is required for proteolytic degradation in autolysosomes. Autophagy 7, 1308-1315 (2011).

\section{Acknowledgements}

The authors are grateful to I. Hanson for editing work. This work was supported by the following grants to H.Z.: 92054301,31630048 and 31790403 from the National Natural Science Foundation of China, 2017YFA0503401 from the Chinese Ministry of Science and Technology, Z181100001318003 from the Beijing Municipal Science and Technology Committee, XDB 19000000 from the Strategic Priority Research Program of the Chinese Academy of Sciences, and OYZDY-SSW-SMC006 from the Key Research Program of Frontier Sciences, Chinese Academy of Sciences. P.C. is supported by grants from the French Agence Nationale pour la Recherche (R18004KK, R16167KK and R18158KK) and Fondation pour la Recherche Médicale.

\section{Author contributions}

The authors contributed equally to all aspects of the article.

\section{Competing interests}

The authors declare no competing interests.

\section{Peer review information}

Nature Reviews Molecular Cell Biology thanks E. Eskelinen, $\mathrm{N}$. Mizushima and $\mathrm{H}$. Nakatogawa for their contribution to the peer review of this work.

\section{Publisher's note}

Springer Nature remains neutral with regard to jurisdictional claims in published maps and institutional affiliations.

(C) Springer Nature Limited 2021 